\title{
Numerical approach to study bubbles and drops evolving through complex geometries by using a level set - moving mesh - immersed boundary method
}

\author{
E. Gutiérrez ${ }^{\mathrm{a}, \mathrm{b}}$, F. Favre ${ }^{\mathrm{c}}$, N. Balcázar ${ }^{\mathrm{a}, \mathrm{b}}$, A. Amani ${ }^{\mathrm{a}}$, J. Rigola $^{\mathrm{a}}$ \\ ${ }^{a}$ Heat and Mass Transfer Technological Center (CTTC), Universitat Politècnica de Catalunya - BarcelonaTech (UPC) \\ ESEIAAT, Colom 11, 08222 Terrassa (Barcelona), Spain \\ ${ }^{b}$ Termo Fluids S.L., Avda Jacquard 97 1-E, 08222 Terrassa (Barcelona), Spain \\ ${ }^{c}$ Instituto de Ingeniería Mecánica y Producción Industrial (IIMPI), Universidad de la República, Uruguay
}

\begin{abstract}
The present work proposes a method to study problems of drops and bubbles evolving in complex geometries.

First, a conservative level set (CLS) method is enforced to handle the multiphase domain while keeping the mass conservation under control. An Arbitrary Lagrangian-Eulerian (ALE) formulation is proposed to optimize the simulation domain. Thus, a moving mesh (MM) will follow the motion of the bubble, allowing the reduction of the computational domain size and the improvement of the mesh quality. This has a direct impact on the computational resources consumption which is notably reduced. Finally, the use of an Immersed Boundary (IB) method allows to deal with intricate geometries and to reproduce internal boundaries within an ALE framework. The resulting method is capable of dealing with full unstructured meshes. Different problems have been studied to assert the proposed formulation, both involving constricting and non-constricting geometries. In particular, the following problems have been addressed: a 2D gravitydriven bubble interacting with a highly-inclined plane, a 2D gravity-driven Taylor bubble turning into a curved channel, the 3D passage of a drop through a periodically constricted channel, and the impingement of a $3 \mathrm{D}$ drop on a flat plate. Good agreement was found for all these cases study, which proves the suitability of the proposed CLS+MM+IB method to study this type of problems.
\end{abstract}

Keywords: Complex geometries, Arbitrary Lagrangian-Eulerian formulation, Level set method, Immersed boundary method, Multiphase flow, Unstructured meshes

\section{Introduction}

The motion of drops and bubbles in complex geometries is of fundamental importance in many scientific and engineering applications. To cite a few examples, chemical reactors generally involve many drop-wall collision processes [1, and its understanding could seriously determine the efficiency of the reactor. The field of microfuidics [2] and lab-on-a-chip concept are fed from the knowledge of the behaviour of bubbles and drops evolving through microgeometries. Additionally, the oil extraction processes could ultimately be reduced to the evolution of a slug flow through constricting solids. 
The motion of bubbles and drops in unbound mediums has attracted significant scientific attention in the last decades (see Tryggvason et al. 3] for an extensive numerical review). On the contrary, the literature about bubbles/drops evolving through complex geometries is far more limited. An meaningful distinction within these problems is stressed here, depending on the relation between the secondary phase and the surrounding geometry. On the one hand, the solid could constrict the bubble or drop, and its trajectory is somehow predetermined by the own shape of the solid. On the other hand, the bubble/drop could freely evolve in an unbounded media, whereas the present solids alter its motion, but in an unconstrained manner. The border between both types of cases is diffuse, and the classification of a specific problem in one group or the other can be ambiguous. See Fig. 1 for a graphical interpretation of both types of problems.

In order to face both types of problems, different approaches have been proposed in the literature. Experimental procedures usually isolate the basic phenomenon to macroscopically study the motion of the secondary phase. See [4, 5, 6, 7] for some valuable experimental works. Additionally, the problem of drops or bubbles evolving in complex geometries can also be addressed theoretically by simplifying the governing equations to extract analytical conclusions (see e.g. 8, 9]). Finally, some valuable numerical approaches have been conducted to solve the aforementioned problem [10, 11, 12, 13. Tab. 1 compiles some of the outstanding works present in the literature, highlighting the method used to solve the problem and the relationship between drop/bubble and geometry.

When facing this type of problems by using a numerical approach, three paramount issues should be addressed in order to satisfactorily solve the case study:

(i) The fluid interface must be computed accurately while conserving integral properties.

(ii) The computational cost should be kept within reasonable bounds.

(iii) The solid geometries, which could be complex and intricate, should be represented effectively and robustly.

Regarding the first item, there are two main groups of methods to deal with multiphase domains. On the one hand, the interface between fluids could be reproduced by using a Front-Tracking method [24]. These techniques accurately describe the multiphase flow, although their implementation may be burdensome due to the need of recomputing the mesh at each time step. On the other hand, the eulerian methods represent the multiphase domain by a continuous (though sharp) change of properties. Those methods include volume-offluid (VoF) techniques [25, level set (LS) methods [26, 27] and hybrid procedures (CLSVOF) [28]. Level set approaches have the advantage of precisely calculating the geometrical properties of the interface (i.e. normal and curvature). However, they present mass conservation drawbacks. On the contrary, the volume-of-fluid methods inherently conserve mass, but at the expense of a troublesome process of computing geometrical properties of the interface. Hybrid methods solve the two issues present in the above-mentioned techniques, but the computational cost significantly increases. In the present work, we propose a methodology based 


\begin{tabular}{|c|c|c|c|}
\hline Reference (year) & Nature of study & $\begin{array}{l}\text { Constrained or } \\
\text { unconstrained }\end{array}$ & Problem description \\
\hline Worthington [5] (1876) & Experimental & Unconstrained & $\begin{array}{l}\text { Qualitative analysis of the impact of } \\
\text { a gravity-driven falling drop against a } \\
\text { horizontal plane }\end{array}$ \\
\hline $\begin{array}{l}\text { Hemmat and Borhan [4] } \\
(1996)\end{array}$ & Experimental & Constrained & $\begin{array}{l}\text { Motion of buoyancy-driven drops } \\
\text { in periodically constricted tubes }\end{array}$ \\
\hline $\begin{array}{l}\text { Hills and Chèty } 14 \text { ] } \\
\text { (1998) }\end{array}$ & Experimental & Constrained & $\begin{array}{l}\text { Gravity-driven rising Taylor bubble } \\
\text { in a concentric annulus tube }\end{array}$ \\
\hline Zhao et al. 15. (1998) & 2D Numerical (LS) & Constrained & $\begin{array}{l}\text { Gravity-driven drop flowing } \\
\text { through an asymmetric funnel }\end{array}$ \\
\hline Aleinov et al. [16] (1999) & $\begin{array}{l}\text { 3D axisymmetric } \\
\text { Numeric (LS) }\end{array}$ & Constrained & $\begin{array}{l}\text { Ejection of a ink drop from } \\
\text { a constricting nozzel. }\end{array}$ \\
\hline $\begin{array}{l}\text { Klaseboer et al. [8] } \\
(2001)\end{array}$ & $\begin{array}{l}\text { Experimental and } \\
\text { theoretical }\end{array}$ & Unconstrained & $\begin{array}{l}\text { Gravity-driven rising bubble } \\
\text { impinging on a horizontal plate }\end{array}$ \\
\hline Olgac et al. 17] (2006) & $\begin{array}{l}\text { 3D axisymmetric } \\
\text { Numeric }(\mathrm{FT})\end{array}$ & Constrained & $\begin{array}{l}\text { Motion of buoyancy-driven drops } \\
\text { in periodically constricted tubes }\end{array}$ \\
\hline Podvin et al. 9] (2008) & $\begin{array}{l}\text { Experimental and } \\
\text { theoretical }\end{array}$ & Unconstrained & $\begin{array}{l}\text { Interaction of a bubble with a } \\
\text { plane at different inclinations }\end{array}$ \\
\hline Deen et al. [10] (2009) & 3D Numerical (FT) & Unconstrained & $\begin{array}{l}\text { Bubbles impacting against a } \\
\text { single sphere or an array of them }\end{array}$ \\
\hline Protière et al. [18 (2010) & Experimental & Constrained & $\begin{array}{l}\text { Passage of a } 2 \mathrm{D} \text { Taylor bubble } \\
\text { through a cylindrical obstacle }\end{array}$ \\
\hline Roudet et al. [19] (2011) & Experimental & Constrained & $\begin{array}{l}\text { Pressure-driven slug flow in meandering } \\
\text { millimetric square channels }\end{array}$ \\
\hline Pozrikidis [20] (2012) & 2D Numerical (FT) & Constrained & $\begin{array}{l}\text { Passage of a viscous liquid through } \\
\text { a bifurcation }\end{array}$ \\
\hline Liu et al. [6] (2015) & $\begin{array}{l}\text { Experimental and } \\
3 \mathrm{D} \text { numerical (LB) }\end{array}$ & Unconstrained & Drop falling against curved surfaces \\
\hline Maitra et al. [7] (2014) & Experimental & Unconstrained & $\begin{array}{l}\text { Drops impacting superhydrophobic } \\
\text { textures }\end{array}$ \\
\hline Gupta et al.[11] (2014) & 2D Numerical (LB) & Unconstrained & $\begin{array}{l}\text { Squeezing mechanism in a } \\
\text { droplet formation device }\end{array}$ \\
\hline Misra et al. 21] (2016) & $\begin{array}{l}\text { 3D axisymmetric } \\
\text { Numeric }(\mathrm{VoF})\end{array}$ & Constrained & $\begin{array}{l}\text { Gravity-driven rising bubble passing } \\
\text { through a circular orifice }\end{array}$ \\
\hline $\begin{array}{l}\text { Izbassarov and } \\
\text { Muradoglu 22] (2016) }\end{array}$ & $\begin{array}{l}\text { 3D axisymmetric } \\
\text { Numeric }(\mathrm{FT})\end{array}$ & Constrained & $\begin{array}{l}\text { Pressure-driven drop through } \\
\text { a sudden contraction and expansion }\end{array}$ \\
\hline $\begin{array}{l}\text { Baltussen et al. } 23 \text { ] } \\
\text { (2017) }\end{array}$ & 3D Numerical (VoF) & Unconstrained & $\begin{array}{l}\text { Interaction of a gravity-driven } \\
\text { rising bubble with a cylindrical solid }\end{array}$ \\
\hline
\end{tabular}

Table 1: Literature summary for bubbles/drops motion through complex geometries, ordered by year of publication. Here "LS" refers to "Level Set method", "FT" to "Front-Tracking method", "LB" to "Lattice-Boltzmann method", and "VoF" to "Volume of Fluids method". 
on a conservative level set (CLS) formulation for unstructured meshes, first reported by Balcázar et al. [29]. The CLS formulation dramatically reduces the mass conservation error in comparison with a standard level set method. This technique has been thoroughly verified [30, 31].

Further efforts have been reported in the development of conservative level-set methods, e.g. the level set remedy approach based on sigmoid function [32], and the accurate conservative level-set method [33]. In the present CLS formulation 29, interface normals are computed using a least-squares method on a wide and symmetric nodes-stencil around the vertexes of the current cell [29]. These normals are then used for an accurate computation of surface tension, without additional reconstruction of the distance function, as in geometrical volume-of-fluid/level-set methods [28 or fast-marching methods [33. Moreover, most computational operations are local. Therefore this method is efficiently implemented for parallel platforms 29, 34. The CLS method has been designed for general unstructured meshes 29. Indeed, the grid can be adapted to any domain, enabling for an efficient mesh distribution in regions where interface resolution has to be maximized 28, 29, 31, 34, 35, which is difficult by using structured grids. Furthermore, a TVD fluxlimiter scheme [29] is used to advect the CLS function, avoiding numerical oscillations around discontinuities, whereas the numerical diffusion is minimized. Finally, the present finite-volume formulation is attractive due to its simplicity and the satisfaction of the integral forms of the conservation laws over the entire domain [29].

When facing the problem of a bubble/drop evolving in complex geometries by using DNS methodologies, the computational resources consumption should be a topic of major concern. This is because the need of enough resolution to represent real geometries, together with the high-demanding process of solving the Navier-Stokes equations. With the exception of basic configurations, a decision should be taken regarding this point. An option is to work under a 2D or axisymmetric hypothesis [11, 22]. However, if a full 3D approach is sought, a domain optimization method becomes mandatory (e.g. non-inertial reference frame, periodic domain, etc.). In the present work, we enforce a moving mesh (MM) technique to deal with small simulation domains. This Arbitrary Lagrangian-Eulerian (ALE) formulation is based upon the work of Estruch et al. 36. The mesh follows the motion of the bubble/drop. Under those circumstances, the simulation domain can be limited to the important regions of the problem (i.e. the bubble/drop and its surroundings). This allows a great saving of computational effort, together with other benefits (see Sec. 4). The drawback of this technique is introducing open boundary conditions at the domain limits, hindering the enforcement of solid conditions. The synergy with the immersed boundary method (introduced below) provides a workable solution to this inconvenience.

In the past few years the Immersed Boundary (IB) method has gained a special interest as an alternative to the body-conformal mesh methods. The IB methods highly simplify the mesh generation process, as they allow the use of Cartesian meshes and the inclusion of moving and/or deforming bodies. In the present framework the IB method constitutes an efficient and simple way to include solid boundaries in the DNS simulations. The boundary condition is imposed by the modification of the discretized Navier-Stokes 
equations, generally by including a forcing term. Depending on how this forcing term is defined, the IB method is classified into two categories [37]: continuous forcing approach and the discrete forcing approach. The original IB method introduced by Peskin [38] in 1972 constitutes a continuous approach. In that work, the immersed boundary is represented by a series of Lagrangian markers linked by springs, which exert a singular force on the fluid by a discrete approximation to the Dirac delta function. This approach has been applied to numerous problems, e.g. biological flows with elastic boundaries [38, 39, 40, 41, It has also been used to represent rigid boundaries by increasing the stiffness of the body [42. However, this approach can lead to stability problems. The discrete forcing approach was introduced by Mohd-Yusof [43] in a spectral method and applied by Fadlun et al. [44] using a finite difference approach. In this case, the forcing is defined in the discrete space by imposing the boundary condition in the solution. This process can be seen as a reconstruction procedure. In fact, in [44] the forcing is not evaluated explicitly. Numerous variants of this approach have been proposed, such as the direct forcing approach [45, 46, the ghost cell method [47, 48] and the Cartesian cut-cell method [49, 50]. The discrete forcing approach allows a sharp representation of the immersed boundary, and is well suited for rigid boundaries. For these reasons, a discrete forcing approach has been taken in the present work.

When an IB method is combined with a CLS method, the mass fluxes at the faces in the vicinity of the solid must be carefully computed to have an accurate convective term, and to ensure that the bubble/drop does not penetrate into the solid regions. The IB method is based on a velocity field reconstruction. However, due to the nature of the fractional step method, the boundary condition is imposed to the predictor velocity. Therefore, an error is made as a result of performing the projection step after imposing the non-slip boundary condition. In this methodology, the solid boundaries are not impermeable and mass flux can pass through them. Thus, in the present formulation this undesirable effect is avoided by imposing a boundary condition to the pressure field using a cut cell based discretization [50, 51] only to the Poisson equation as in [52].

In light of the foregoing, the present work proposes a new insight to face problems where a bubble or drop is evolving through an arbitrary geometry (constricting or non-constricting). The method is a combination of a CLS technique to deal with the multiphase domain, an ALE framework to optimize the size of the simulation domain, and an IB method to represent the embedded solids. To the best of our knowledge, this paper is the first work combining a CLS technique, a dynamic mesh framework and an IB method. The resulting method is capable of dealing with full unstructured meshes, which greatly increases the versatility of this methodology.

The paper is organized as follows: Sec 2 presents the mathematical description of the proposed CLS+MM+IB method. The numerical treatment of these equations is then addressed through Sec. 3 , followed by a discussion on the applicability of the method (Sec. 4). Then, results of the validation and verification cases are presented in Sec. 5. Finally, conclusions are summarized in Sec. 6. 


\section{Mathematical formulation}

In the present section, the mathematical foundation of the proposed CLS+MM+IB method to solve multiphase flows in complex geometries is presented. The equations to be solved are the Navier-Stokes equations with the hypotheses of incompressible flow, Newtonian fluids, no mass transfer at the fluids interface, Arbitrary Lagrangian-Eulerian framework, constant surface tension coefficient $\sigma$ and embedded solids. Those equations are given by the conservation laws of mass and momentum, as follows:

$$
\begin{gathered}
\nabla \cdot \mathbf{v}=0 \\
\frac{\partial}{\partial t}(\rho \mathbf{v})+\nabla \cdot\left(\rho \mathbf{v}\left(\mathbf{v}-\mathbf{v}_{\text {domain }}\right)\right)=-\nabla p+\nabla \cdot \mu\left(\nabla \mathbf{v}+(\nabla \mathbf{v})^{T}\right)+\rho \mathbf{g}+\sigma \kappa \mathbf{n} \delta_{\Gamma}+\Psi_{\mathrm{IB}}
\end{gathered}
$$

where $t$ is the time, $\mathbf{v}$ is the velocity vector, $\mathbf{v}_{\text {domain }}$ is the domain velocity, $p$ is the pressure, $\mathbf{g}$ is the gravity acceleration, $\sigma$ is the constant surface tension coefficient, $\kappa$ and $\mathbf{n}$ are respectively the curvature and the unit normal vector of the interface between fluids, $\delta_{\Gamma}$ is the Dirac delta function located at that interface, and $\Psi_{\mathrm{IB}}$ is an extra source term introduced by the immersed boundary method (see Sec. 3.2. Finally, $\rho$ and $\mu$ are the

fluid density and viscosity, respectively. These properties are constant within each fluid. Thus, they can be expressed as a single property with a jump discontinuity at the interface, yielding the following equations:

$$
\begin{gathered}
\rho=\rho_{1} H+\rho_{2}(1-H) \\
\mu=\mu_{1} H+\mu_{2}(1-H)
\end{gathered}
$$

Subscripts 1 and 2 refer to the suspending fluid and secondary phase, respectively; and $H$ is the Heaviside function with its discontinuity located at the fluids interface. $H$ is equal to zero in the secondary phase, and equal to 1 in the suspending fluid.

\subsection{Interface capturing}

Equations from 1 to 4 close a non-continuous problem, due to the jump of properties located at the interface and the surface tension source term of the momentum equation (Eq.2) acting only at that interface. However, aiming to avoid numerical instabilities at the interface, a continuous treatment of the fluids properties is more convenient. A conservative level set (CLS) method, as introduced by [29] in the context of unstructured grids, is used to tackle with the fluid interface. Therefore, the interface is implicitly represented by an indicator function $\phi$, defined as:

$$
\phi(\mathbf{x}, t)=\frac{1}{2}\left(\tanh \left(\frac{d(\mathbf{x}, t)}{2 \varepsilon}\right)+1\right)
$$

Here $d(\mathbf{x}, t)$ is the signed distance function, and $\varepsilon$ is a parameter to control the thickness of the interface between fluids. This interface can be located by obtaining the $\phi=0.5$ isosurface. 
By means of the level set function, Eqs. 3 and 4 can be expressed as follows:

$$
\begin{aligned}
& \rho=\rho_{1} \phi+\rho_{2}(1-\phi) \\
& \mu=\mu_{1} \phi+\mu_{2}(1-\phi)
\end{aligned}
$$

The solution of the Navier-Stoke equations (Eqs. 1 and 2) provides the velocity field $\mathbf{v}$ used to advect $\phi$. This transport equation can be written as follows:

$$
\frac{\partial \phi}{\partial t}+\nabla \cdot \phi\left(\mathbf{v}-\mathbf{v}_{\text {domain }}\right)=0
$$

After advection, a reinitialization step is needed to complete the calculus of the level set function. In this step, the interface is compressed seeking to maintain a constant thickness, as it tends to widen in the advection process due to numerical diffusion [53. Therefore, the following differential equation is computed:

$$
\frac{\partial \phi}{\partial \tau}+\nabla \cdot \phi(1-\phi) \mathbf{n}_{\tau=0}=\nabla \cdot \varepsilon \nabla \phi
$$

This equation is advected in pseudo-time $\tau$. On the one hand, the term $\phi(1-\phi) \mathbf{n}_{\tau=0}$ compresses the level set function along the unit normal vector $\mathbf{n}$, aiming to sharpen the interface. On the other hand, the diffusive term $\nabla \cdot \varepsilon \nabla \phi$ ensures that the characteristic thickness of the profile is proportional to $\varepsilon=0.5 h^{0.9}$, where $h$ is the grid size computed as the cubic root of the cell volume [29].

\subsection{Surface tension treatment}

By implementing an appropriate surface tension model, a twofold goal is sought. First, the calculus of the curvature $\kappa$, and second, the application of the pressure jump to the fluid domain. With this aim, a CSF model [54] has been adopted. This technique enables the conversion of the singular term $\sigma \kappa \mathbf{n} \delta_{\Gamma}$ into a volume force:

$$
\sigma \kappa \mathbf{n} \delta_{\Gamma}=\sigma \kappa(\phi) \nabla \phi
$$

where $\kappa(\phi)$ and $\mathbf{n}$ are given by:

$$
\begin{gathered}
\kappa(\phi)=-\nabla \cdot \mathbf{n} \\
\mathbf{n}=\frac{\nabla \phi}{\|\nabla \phi\|}
\end{gathered}
$$

Here, $\nabla \phi$ is computed by means of a least-square method [29].

\section{Numerical solution}

The set of equations posed above has been discretized onto a collocated grid arrangement. A finite-volume method has been enforced, according to [29]. The diffusion terms of the governing equations (Eqs. 2]and 9p are 
discretized by using a Central Difference (CD) scheme. In addition, a superbee flux limiter for unstructured meshes [29] is applied to the convective term of the advection equation (Eq. 8), and a CD scheme is used for the convective term of the momentum equation (Eq. 2). For time discretization, a 3-step-third-order accurate TDV Runge-Kutta scheme [55] is enforced in the advection and reinitialization equations (Eqs. 8 and 95. Finally, both compressive and diffusive terms of the reinitialization equation (Eq. 9) are discretized by using a CD scheme 29].

The pressure-velocity coupling is solved by means of a Fractional Step method [56, 57, 29, 58. Momentum equation (Eq. 2) is computed in two steps:

$$
\frac{\rho \mathbf{v}^{\mathrm{p}}-\rho^{n} \mathbf{v}^{n}}{\Delta t}=\nabla \cdot\left[\rho \mathbf{v}^{n}\left(\mathbf{v}^{n}-\mathbf{v}_{\text {domain }}^{n}\right)\right]+\nabla \cdot \mu\left(\nabla \mathbf{v}^{n}+\nabla^{T} \mathbf{v}^{n}\right)+\rho \mathbf{g}+\sigma \kappa \nabla \phi+\Psi_{\mathrm{IB}}
$$

For the sake of simplicity, this equation has been discretized using an explicit Euler scheme, although an explicit Adams-Bashforth scheme has been used for computations. Superscript "p" refers to predictor variables, $n$ to the current time step, and $n+1$ to the following one. The next step of the method is the calculation of the following expression:

$$
\mathbf{v}^{n+1}=\mathbf{v}^{\mathrm{p}}-\frac{\Delta t}{\rho} \nabla p^{n+1}
$$

By adding the continuity equation (Eq. 1), the following Poisson equation is obtained to solve the pressure field:

$$
\nabla \cdot\left(\frac{1}{\rho} \nabla p^{n+1}\right)=\frac{1}{\Delta t} \nabla \cdot \mathbf{v}^{\mathrm{p}}
$$

The discretization of this equation leads to a linear system, which is solved by means of a preconditioned conjugate gradient method. Cell-face velocity is calculated according to [29, 58, in order to avoid pressurevelocity decoupling and to fulfill the incompressible constraint. This cell-face velocity is used to advect the CLS function in Eq. 8, and momentum in Eq. 2

\subsection{Mesh movement and boundary conditions}

The mesh is moved following the evolution of the bubble/drop. Thus, to an observer standing on the mesh, the bubble/drop will be always around its initial position (although its shape may change). The first consequence of this approach is the need of open boundaries at the domain limits. Consequently, inflow or outflow conditions are imposed at the boundary faces of the fluid domain.

The inflow boundary condition imposes a null velocity vector at the corresponding boundary, and a zero gradient condition for the pressure. The outflow boundary condition is a combination of a convective boundary condition and the formulation proposed by Davis and Moore [59]. The reader is referred to [35] for further notes on the formulation of the outflow boundary condition.

To determine whether a specific boundary face is inflow or outflow, the following dot product is evaluated: $\mathbf{v}_{\mathrm{G}} \cdot \mathbf{n}_{b f}$, where $\mathbf{v}_{\mathrm{G}}$ is the velocity of the bubble/drop centroid $G$ (see App. B), and $\mathbf{n}_{b f}$ is the outward unit normal vector to the boundary face. If the value of this dot product is positive, it means that the bubble/drop 
is moving towards this face. Thus, the boundary face should be an inflow. Otherwise the boundary face under consideration is set as outflow. It is worth emphasizing that both conditions inflow/outflow collapse into the same formulation in the limit case where the aforementioned dot product is close to 0 (see [35]). It is also worth to mention that in cases with long distances between the bubble/drop centroid and the boundaries (where the fluid velocity near the domain limits is close to zero), the treatment of the inflow and outflow becomes less important, and even free-slip conditions could work well (see e.g. [12]).

Additionally, when an Arbitrary Lagrangian-Eulerian approach is adopted, the mass flux through a face $f$ needs to be modified as follows [36:

$$
\dot{m}_{f}^{\text {modified }}=\int_{S_{f}} \rho\left(\mathbf{v}-\mathbf{v}_{S}\right) \cdot \mathbf{n} \mathrm{d} S \approx \rho_{f}(\mathbf{v} \cdot \mathbf{n})_{f} S_{f}-\rho_{f}\left(\mathbf{v}_{S} \cdot \mathbf{n}\right)_{f} S_{f}
$$

where subscript $f$ denotes that the corresponding variable is evaluated at face $f$, and $\mathbf{v}_{S}$ is the surface velocity whose outward unit vector is represented by $\mathbf{n}$. Linear interpolation between nodes is used when a certain variable is unknown at a specific face (e.g. for the density $\rho$ ). The term $\left(\mathbf{v}_{S} \cdot \mathbf{n}\right)_{f} S_{f}$ represents the volume swept by the $\mathrm{CV}$ face $f$ per unit of time. As no deformation is allowed, the movement of the mesh is a combination of a linear translation and a rotation from the bubble/drop centroid $G$. Therefore, the swept volume is computed exactly by evaluating the term $\mathbf{v}_{S}$ at the centroid of the face. Moreover, the cells volumes remain unchanged due to the nature of the mesh movement, which combined with the exact calculation of the swept volume ensure the accomplishment of the so-called Space Conservation Law (SCL) [36]:

$$
\frac{d}{d t} \int_{\Omega_{\mathrm{CV}}} \mathrm{d} \Omega_{\mathrm{CV}}+\int_{S} \mathbf{v}_{S} \cdot \mathbf{n} \mathrm{d} S=0
$$

where $\Omega_{\mathrm{CV}}$ is the volume of the moving cell, and $S$ is its closed surface. Note that the first term of this equation is strictly zero because $\Omega_{\mathrm{CV}}$ is constant, and the second term is also zero because it is computed exactly as explained above. Therefore, the SCL is ensured and the mass conservation is procured.

The definition of the translation and rotation of the mesh is not imposed by the physic, but should be coherent with the evolution of the bubble/drop within the domain. Two main scenarios can be identified (see Fig. 1):

(i) When the bubble/drop is evolving in an unconstrained domain where its trajectory can not be easily predicted in advance. In this case, the linear velocity of the mesh $\mathbf{v}_{\text {domain }}$ is taken equal to the bubble/drop velocity $\mathbf{v}_{\mathrm{G}}$. Additionally, its angular velocity $\boldsymbol{\omega}_{\text {domain }}$ is taken equal to the one that the bubble/drop velocity vector has: $\boldsymbol{\omega}_{\text {domain }}=\frac{\mathrm{d} \alpha}{\mathrm{d} t}$ where $\alpha$ is the angle rotated by the velocity vector of the bubble/drop. The centre of rotation is the bubble/drop centroid $G$.

(ii) When the bubble/drop is evolving in a tube or, in general, in a constricting geometry generated by the extrusion of a curve through a driving curve. Note that the extruded curve could vary along the driving curve. The only requirement is that the driving curve should be unequivocally defined. In this case, the 
linear velocity of the mesh is taken equal to the projection of the bubble/drop velocity onto the driving curve. Consequently, $\mathbf{v}_{\text {domain }}=\left(\mathbf{v}_{\mathrm{G}} \cdot \hat{\mathbf{n}}_{\mathbf{t}}\right) \hat{\mathbf{n}}_{\mathbf{t}}$, where $\hat{\mathbf{n}}_{\mathbf{t}}$ is the tangent vector of the driving curve at the projection of the centroid of the bubble/drop $G$ onto the driving curve (namely $G^{\prime}$ ). In addition, the angular velocity of the domain $\boldsymbol{\omega}_{\text {domain }}$ is equal to the one that the tangent vector $\hat{\mathbf{n}}_{\mathbf{t}}$ of the driving curve has: $\boldsymbol{\omega}_{\text {domain }}=\frac{\mathrm{d} \hat{\mathbf{n}}_{t}}{\mathrm{~d} t}$. The centre of rotation is the point $G^{\prime}$. Fig. 2 sketches this geometrical configuration.

The first approach described above should be able to tackle the second situation. However, the distinction seems desirable in order to get a more natural movement of the mesh in cases with a constricting geometry. It is also intended to avoid an instance where the mesh does not cover the whole cross section of the pipe. See Sec. 5.2 for cases with both implementations. Regarding the second scenario, there are some further considerations to be taken into account. The formulation presented above assumes the analytical description of the driving curve as known. In some situations (e.g. in industrial applications) this issue constitutes a limitation, as the tubular geometry of the problem might not be analytically described. In this case, two alternatives are proposed: the use of the general approach for unconstrained geometries, and the calculation of a "predicted" driving curve by interpolation between points of the tubular geometry. An additional problem is establishing the shape and the dimensions of the fluid domain. The longitudinal length is set by the physics of the problem (see [35]). However, the lateral distance must be kept as small as possible in order to save resources, but it should be enough to cover the whole transversal section of the tube during the whole simulation. There is no straightforward solution for this issue, although it is analytically approachable for easy geometries (see e.g. the case presented in Sec. 5.2 .

\subsection{Immersed boundary treatment}

The forcing term $\Psi_{\mathrm{IB}}$ in Eq. 13 is included to enforce the non-slip immersed boundary condition. It affects the nodes in the vicinity and the interior of the immersed bodies. If the solid moves with respect to the mesh, the nodes are classified at every iteration into three categories: interior, forcing and exterior points (see Fig. 3). The interior points are those falling within the immersed body. The forcing points are those outside the solid, which meet one or both of these conditions: (i) the node has a neighbour which is inside the object, (ii) their cell-volume is cut by the immersed boundary. The remaining nodes are the so-called exterior points.

To classify the nodes, a signed distance field $\varphi(\mathbf{x}, t)$ is defined as the minimum distance from the node position to the immersed boundary. In order to be able to handle any geometry, the immersed boundary is represented by an unstructured surface mesh, composed by a series of triangles in stereo-lithography (STL) format [60]. To calculate the minimum distance between a particular node of the mesh to the Immersed Boundary, the closest triangle is found and the closest point of that triangle is computed. Finally, the sign of the distance is defined using the outpointing normal vector of that particular triangle, given positive values 


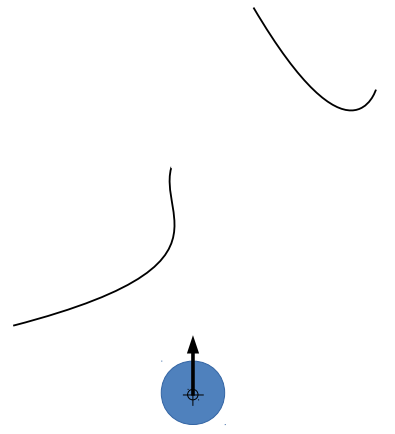

(a)

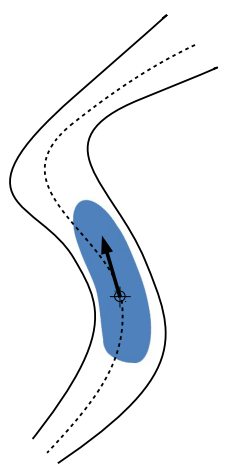

(b)

Figure 1: Two main scenarios may appear when studying the evolution of bubbles/drops in complex geometries: (a) an unconstrained situation, in which the geometry does not determine beforehand the movement of the bubble/drop; and (b) a constrained situation where a tubular geometry forces the movement of the bubble/drop following a driving curve.

for internal nodes and negative for the external ones.

Once the nodes have been classified, the source term is calculated. This source term is zero in the exterior points, and it is evaluated with the following expression for the rest of the nodes 61]:

$$
\Psi_{\mathrm{IB}}=\frac{\rho \mathbf{V}-\rho \mathbf{v}^{n}}{\Delta t}-\left\{\nabla \cdot\left[\rho \mathbf{v}^{n}\left(\mathbf{v}^{n}-\mathbf{v}_{\text {domain }}^{n}\right)\right]+\nabla \cdot \mu\left(\nabla \mathbf{v}^{n}+\nabla^{T} \mathbf{v}^{n}\right)+\rho \mathbf{g}+\sigma \kappa \nabla \phi\right\}
$$

where $\mathbf{V}$ is the desired value of the velocity field. For a prescribed movement of the body, the velocities $\mathrm{V}$ of the interior points are directly calculated from their coordinates. However, in order to compute the velocities of the forcing points some approximations have to be used, as by definition these nodes are outside the object. In this particular case, $\mathbf{V}$ is computed by means of a second-order interpolation among the local velocity of the solid, and the predictor velocity $\mathbf{v}_{\Psi_{\mathrm{IB}}=0}^{\mathrm{p}}$ of neighbour nodes calculated when $\Psi_{\mathrm{IB}}=0$. The reader is referred to the work by Favre et al. 61 for further details about the calculation of $\Psi_{\text {IB }}$.

Due to the nature of the fractional step method, in the present formulation of the IB method the boundary condition is imposed to the predictor velocity. Some inaccuracy is introduced in the solution, because the projection step is performed after imposing the non-slip boundary condition. As a result of this procedure, some mass flux may pass through the solid boundaries, which does not affect the accuracy of the solution 


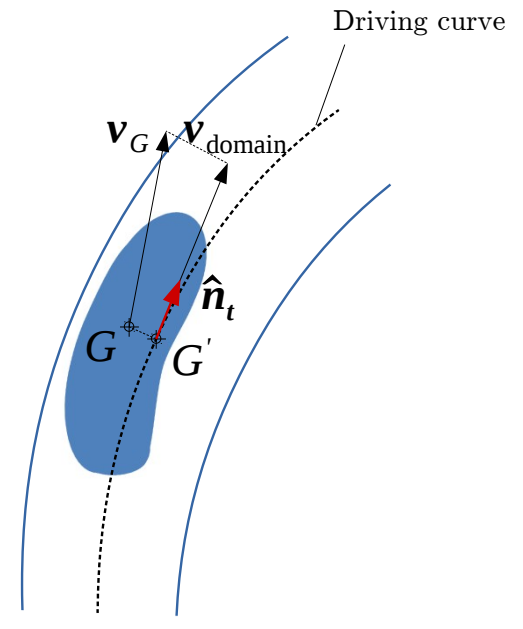

Figure 2: Geometrical objects needed to define the mesh movement in problems with a constricting geometry. $G$ is the bubble/drop centroid, $G^{\prime}$ is its projection onto the driving curve of the pipe, $\mathbf{v}_{G}$ is the velocity of the bubble/drop centroid, $\hat{\mathbf{n}}_{\mathbf{t}}$ is the unit tangent vector of the driving curve at $G^{\prime}$, and $\mathbf{v}_{\text {domain }}$ is the velocity of the computational domain.

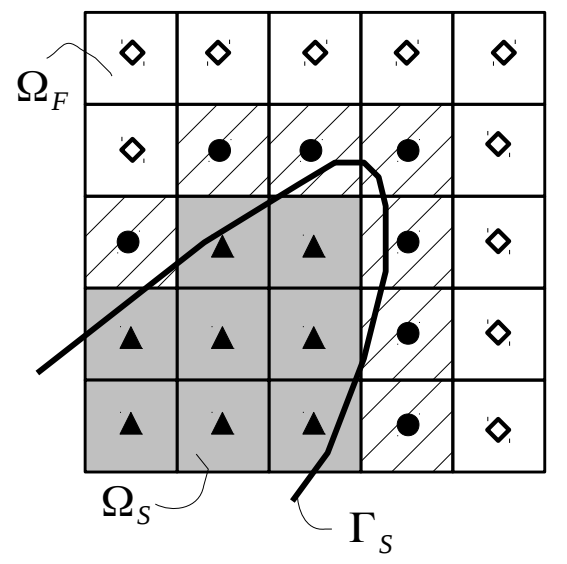

Figure 3: Example of control volumes intersected by an object: $\left(\Omega_{S}\right)$ solid region, $\left(\Omega_{F}\right)$ fluid region, $\left(\Gamma_{S}\right)$ solid-fluid interface. The nodes are classified as: $(\diamond)$ exterior points, $(\bullet)$ forcing points, and $(\boldsymbol{\Delta})$ interior points. 
but can produce unphysical results, e.g. a particular fluid-phase could penetrate into an immersed body. This undesirable effect can be avoided by imposing a boundary condition to the pressure field using a cut-cell based discretization [50, 51] only to the Poisson equation, as in [52]. A cut-cell is a cell with some of its volume at the fluid region and some at the solid region. Two types of cut-cells can be distinguished: those with the node in the fluid region (generally called "regular cut-cells"), and those with the node in the solid (called "small cut-cells", because the fluid fraction of it could be arbitrarily small). Both types of cells are shown in Fig. 4.

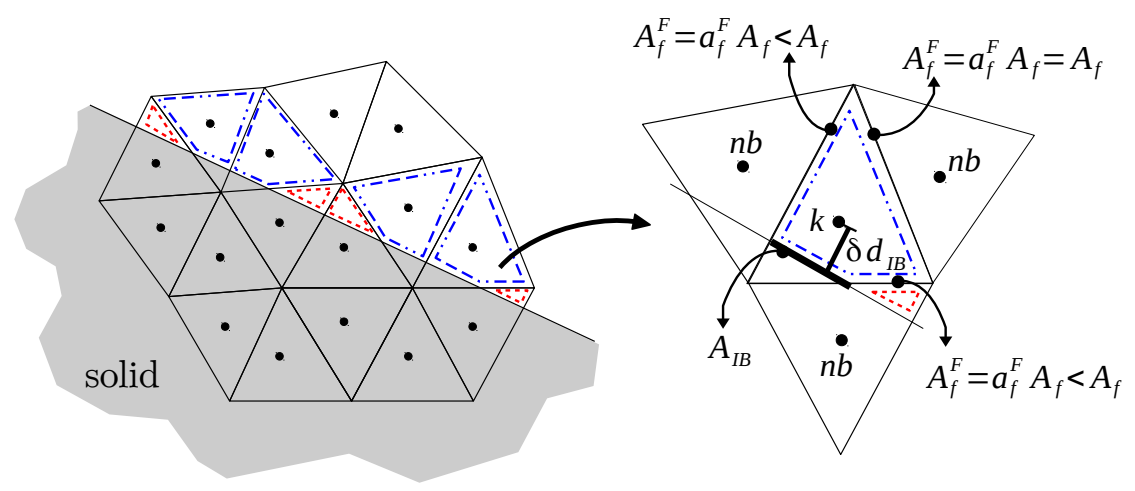

Figure 4: Definition of regular cut-cells and small cut-cells. The blue dot-dash lines denote the regular cut-cells, and the red dash lines mark the small cut-cells. Some of the faces of a cut-cell are divided into a fluid-face and a solid-face, where $A_{f}^{F}$ represents the surface of the fluid-face. The surface of the solid-fluid interface contained within the cut-cell is indicated here as $A_{\mathrm{IB}}$. Finally, $\delta d_{\mathrm{IB}}$ is the distance between the node of the cut-cell and the solid-fluid interface.

The finite-volume discretization of the Poisson equation for a generic cell $k$ is written as:

$$
\begin{aligned}
\dot{m}_{\mathrm{IB}}^{\mathrm{p}}+\sum_{f \in \mathcal{F}(k)} \dot{m}_{f}^{\mathrm{p}} & =\Delta t\left(\left(p_{\mathrm{IB}}^{n+1}-p_{k}^{n+1}\right) \frac{A_{\mathrm{IB}}}{\delta d_{\mathrm{IB}}}+\sum_{f}\left(p_{n b}^{n+1}-p_{k}^{n+1}\right) \frac{a_{f}^{F} A_{f}}{\delta d_{f}}\right) \\
\mathbf{v}_{k}^{n+1} & =\mathbf{v}_{k}^{\mathrm{p}}-\frac{\Delta t}{\rho \gamma V_{k}}\left(p_{\mathrm{IB}}^{n+1} \vec{n}_{\mathrm{IB}}^{F} A_{\mathrm{IB}}+\sum_{f} p_{f}^{n+1} \vec{n}_{f}^{F} a_{f}^{F} A_{f}\right) \\
\dot{m}_{f}^{n+1} & =\dot{m}_{f}^{\mathrm{p}}-\Delta t a_{f}^{F} A_{f} \frac{\left(p_{\mathrm{IB}}^{n+1}-p_{k}^{n+1}\right)}{\delta d_{f}}
\end{aligned}
$$

where the superscript "p" denotes that the corresponding variable is a "predictor" one, $\mathcal{F}(k)$ is the set of the faces of cell $k, a_{f}^{F}=A_{f}^{F} / A_{f}$ is the fluid surface fraction of face $f$ and $\gamma=V_{k}^{F} / V_{k}$ is the volume fraction of 
cell $k$. The subscript IB refers to the section of the immersed boundary intersected by cell $k$ called $\partial \mathrm{IB}, A_{\mathrm{IB}}$ is its surface, $\delta d_{\mathrm{IB}}$ is the distance between the node of $k$ and $\partial \mathrm{IB}$, and $p_{\mathrm{IB}}$ is the pressure at that location. The subscript $n b$ refer to the face-neighbour of $k$, and $\delta d_{f}$ is the normal-projected distance between the nodes $k$ and $n b$. Superscript $F$ in the normal vectors $\mathbf{n}_{\mathrm{IB}}^{F}$ and $\mathbf{n}_{f}^{F}$ indicates that those vectors point to the fluid region. For the cells that are not cut by an immersed boundary, all the coefficients $a_{f}^{F}$ and $\gamma$ are equal to 1. Therefore, the discretization corresponds to the classic second-order spectro-consistent scheme [62]

However, small cells are treated with a different approach. As they can be arbitrarily small, the use of the same approach as for the regular cut-cells could lead to an ill-conditioned pressure Poisson equation. Small cut-cells can also cause viscous stability problems. Therefore, the so-called "virtual cell merging technique" is used in the present work. It was first proposed by Meyer et al. 63] for the momentum equation, and adapted by Seo and Mittal [52] for the pressure Poisson equation. It consists of modifying the independent term of the Poisson equation. First, the independent terms $b(k)=\sum_{f} \dot{m}_{f}^{P}$ are computed for every cell, including the small ones. Thus, the term $b_{\text {small }}$ of each small cell is transferred to its regular neighbours. This means that the term $b_{n b}$ of each regular neighbour is incremented by a fraction of $b_{\text {small }}$. Given a small cell, the fraction of $b_{\text {small }}$ which is transferred to each regular neighbour is proportional to the fluid surface shared with that neighbour. Once this transfer is done, the independent terms of the Poisson equation corresponding to the small cells become zero.

The verification of the current implementation of the IB method is presented in App. A where the problem of a flow around a sphere is solved for different Reynolds numbers.

\subsection{Time step}

In order to obtain a suitable time step at each iteration, a CFL condition is enforced. Therefore, the following condition is obtained by comparison of the different terms of the momentum equation (Eq. 2):

$$
\Delta t=\mathcal{C}_{\mathrm{CFL}} \cdot \min \left(\frac{h}{\left\|\mathbf{v}^{n}\right\|}, \frac{h^{2} \rho^{n}}{\mu^{n}}, \sqrt{\frac{h}{g}},\left(\frac{\rho_{1}+\rho_{2}}{4 \pi \sigma}\right)^{1 / 2} h^{3 / 2}, \frac{h}{\left\|\mathbf{v}_{\text {domain }}\right\|}\right)
$$

where $g=\|\mathbf{g}\|$, and $\mathcal{C}_{\mathrm{CFL}}$ is a safety constant $\left(\mathcal{C}_{\mathrm{CFL}} \approx 0.1\right)$. It is worth noting that the source term from the immersed boundary method (namely, $\Psi_{\mathrm{IB}}$ ) does not additionally restrict the time step. At this point, the effect of the embedded body has already been taken into account in the calculus of the velocity field. Therefore, the convective restriction (first term of the left-hand part of Eq. 222) already includes the effect of the IB method on the time step.

\subsection{Calculation algorithm}

Alg. 11 summarizes the calculation procedure needed to advance from the current time step $t^{m}$ to the next one $t^{m+1}$. This procedure has been implemented in a parallel in-house $\mathrm{c}++/$ MPI code called TermoFlu$i d s$ 64. 


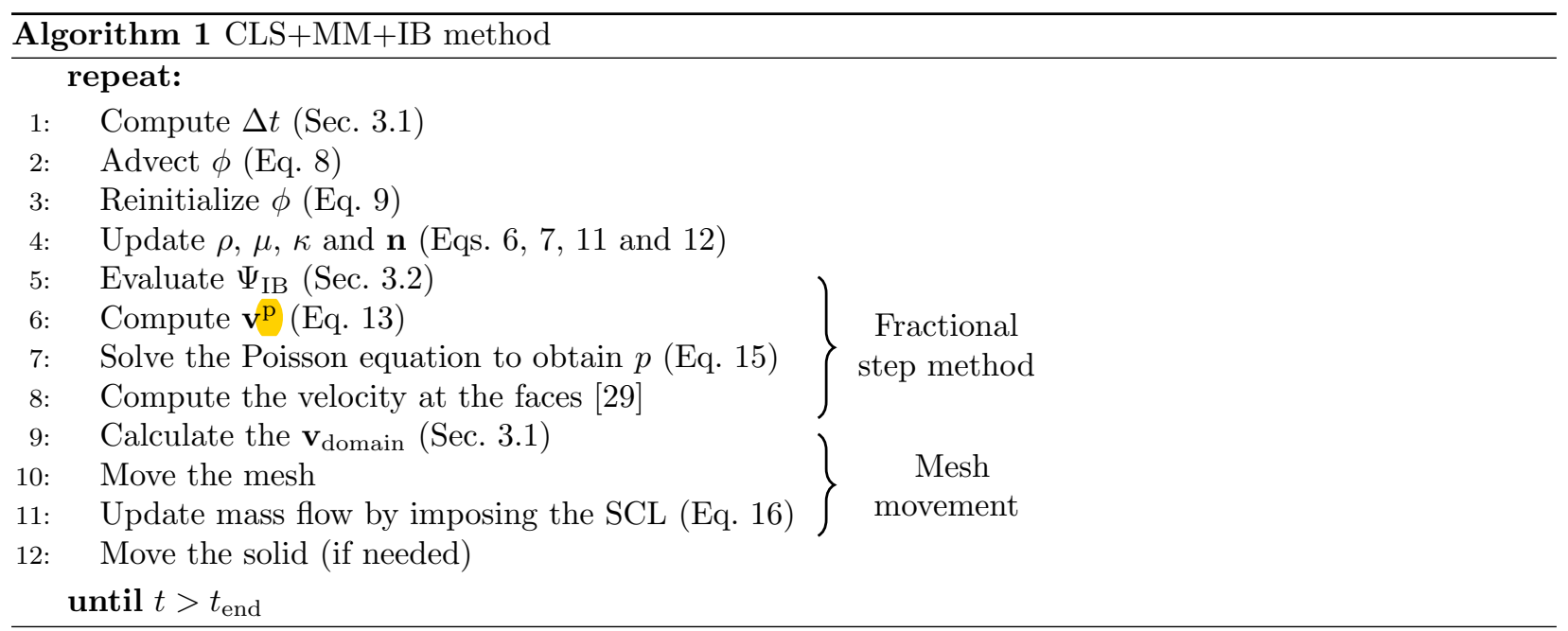

\section{Discussion on the method}

The proposed approach exploits some striking features of the comprising methods. First, the CLS method allows dealing with multiphase domains, while keeping the mass error under control [29]. Second, the dynamic mesh technique enables using small computational domains, covering only the important regions of the problem 35. Finally, using an IB method leads to representing intricate solids which do not conform the mesh shape (see Sec. 3.2). Furthermore, the synergy among those methods brings additional benefits:

- It is possible to reproduce internal boundaries (i.e. solid and free-slip conditions) in an ALE framework working with open boundaries.

- Long temporal evolutions can be studied with no need of increasing the size of the mesh, e.g. problems involving infinity domains (i.e. tubes).

- The mesh quality is homogenised, regardless of the geometry. That avoids the worsening of the mesh quality due to the potential need of reproducing certain intricate geometries.

- The method greatly facilitates on-the-go testing and parametric studies. This is because, in general, a modification of the geometry or flow conditions does not imply a change of the mesh.

- Since the bubble/drop stays steady at a specific location within the computational domain, the mesh can be improved by refining the important regions of the problem (e.g. the interface between fluids). This notably complements the increased versatility achieved by working in a full unstructured framework. In general terms the global benefit is similar to the one obtained by using an adaptive mesh refinement (AMR) technique.

- By using the proposed CLS+MM+IB method, the obtained solution of a specific multiphase problem 
conserves the mass, as all of the consisting methods are mass-conservative (i.e. CLS method [29], ALE methodology 36, 35, and the IB method explained in Sec 3.2.

- The method allows to modify geometrical conditions during the simulation. This is particularly useful when studying the impact of bubbles/drops against objects. In the analysis of these problems, terminal conditions in the motion of the bubble/drop are usually assumed. With the proposed method, by placing the solids out of the fluid domain, free motion conditions are initially applied to the drop/bubble. When this drop/bubble achieves its steady state, the solid is positioned in the appropriate place. Otherwise, the initial distance from the bubble/drop centroid to the solid could not be enough to achieve the terminal velocity, or it could be excessive entailing unnecessary computational expenditure.

There are also some few drawbacks to keep in mind when considering the proposed method:

- The CLS+MM+IB method does not easily deal with multiple bubbles/drops problems, or cases involving break-up. Complications arise in defining the mesh movement, as it is specified based on the velocity of a bubble/drop centroid (see Sec. 3.1). This criterion becomes obsolete in cases with multiple bubbles/drops, and it should be revised. Cases with large deformations could also be problematic, since the drop/bubble might get too close to a domain boundary.

- The boundary condition design becomes troublesome due to the inherent presence of open limits. Both the formulation of these boundaries and their placement within the domain require a careful treatment. See 35] for a detailed discussion on this issue.

- When dealing with constricting geometries, a minimum domain is desirable (although not essential) in order to save computational resources. The domain dimensions should assure that the computational domain covers the whole transversal section of the pipe throughout the complete simulation. However, the sizing of this minimum domain is not straightforward. See Sec. 3.1 for further notes on this topic.

\section{Results and validation}

In the present section, some studies are conducted in order to assert the validity and accurateness of the proposed CLS+MM+IB method presented above. First, in order to capture the particular potentialities of the method, we designed one pair of two-dimensional experiments, which involve complex geometries, mesh translation and mesh rotation. Reference results are obtained by running the same case within a static mesh, and without employing immersed boundaries. This last approach is the usual way to proceed when facing multiphase DNS problems. In these reference cases, although the mesh resolution is kept constant, the fluid domain is the same as the physical domain. Therefore, much bigger meshes are needed. By proceeding this way, the particularities of the presented method can be successfully evaluated in comparison with the general approach for multiphase DNS in complex geometries, providing a consistent way to make measurable 
comparisons. In addition, a clear discussion on the simulation times and the resource consumption can be drawn. After these cases, two three-dimensional problems are presented. By doing so, it is intended to validate the proposed method in full 3D configurations. In these cases, the obtained results are compared with those found in the literature.

\subsection{Two-dimensional gravity-driven bubble approaching an inclined plane}

In this section, we propose the study of the passage of a bouncing bubble through a highly-inclined plane. This case is very convenient to accomplish the goals of the present section, due to the following reasons. First, it involves a clear-cut rotating process which allows the evaluation of the rotational mesh mechanism. Second, the well-defined boundary treatment and a deeply study flow regime [65] allow us to focus attention on the phenomenology of the bubble-wall interaction. Finally, it is affordable to simulate the same problem with the complete domain, without moving the mesh or using immersed boundaries. This is very useful for the sake of comparison, as differences in the obtained results can be attributed exclusively to particularities of the proposed CLS+MM+IB method. This comparison technique has been broadly used in CFD, e.g. when validating a new LES formulation against a DNS case.

Therefore, the problem has been solved by using two methods: the proposed CLS+MM+IB method and a traditional approach, with the complete domain and ordinary boundaries. Fig. 5 sketches the geometrical configuration and initial set up of both cases, showing the distances from the bubble to the boundaries. It is worth noting that an increased left lateral distance is needed for the case of moving mesh, as the bubble could move beyond the initial left distance to the wall after bouncing (and in fact it does). Distances from the bubble to the inflow and outflow when solving the problem with the proposed CLS+MM+IB method are overestimated in comparison with those obtained with the method proposed in [35. Thus, a smaller domain would actually be capable of capturing the physics involved in the problem. However, with the aim of minimizing the discrepancies among methods caused by this reason, bigger distances were chosen.

Physical properties are determined by the following dimensionless numbers:

$$
\eta_{\rho}=\frac{\rho_{1}}{\rho_{2}} \quad, \quad \eta_{\mu}=\frac{\mu_{1}}{\mu_{2}} \quad, \quad E o=\frac{g d^{2}\left(\rho_{1}-\rho_{2}\right)}{\sigma} \quad, \quad M o=\frac{g \mu_{1}^{4}\left(\rho_{1}-\rho_{2}\right)}{\rho_{1}^{2} \sigma^{3}}
$$

where $d$ is the initial bubble diameter, and $\eta_{\rho}$ and $\eta_{\mu}$ are the density and viscosity ratios, respectively. Eo is the Eötvös number and $M o$ is the Morton number. In the present problem, $\eta_{\rho}=10, \eta_{\mu}=10, E o=9.0$ and $M o=6 \cdot 10^{-4}$. This flow regime coincides with that of the well-known benchmark case first studied in 65.

In both methodologies, the employed meshes are full unstructured and composed of triangular control volumes. In order to ensure the mesh independence of the obtained results, several cell sizes $h$ are tested. In particular, three different meshes are used for both the case with full domain and the one with moving mesh. The chosen resolutions are $d / 15, d / 23$ and $d / 30$. Therefore, the meshes are labelled from $M 1$ to $M 3$ in increasing order of resolution, adding the subscript "FD" for the full domain case, and "MM" for the moving 


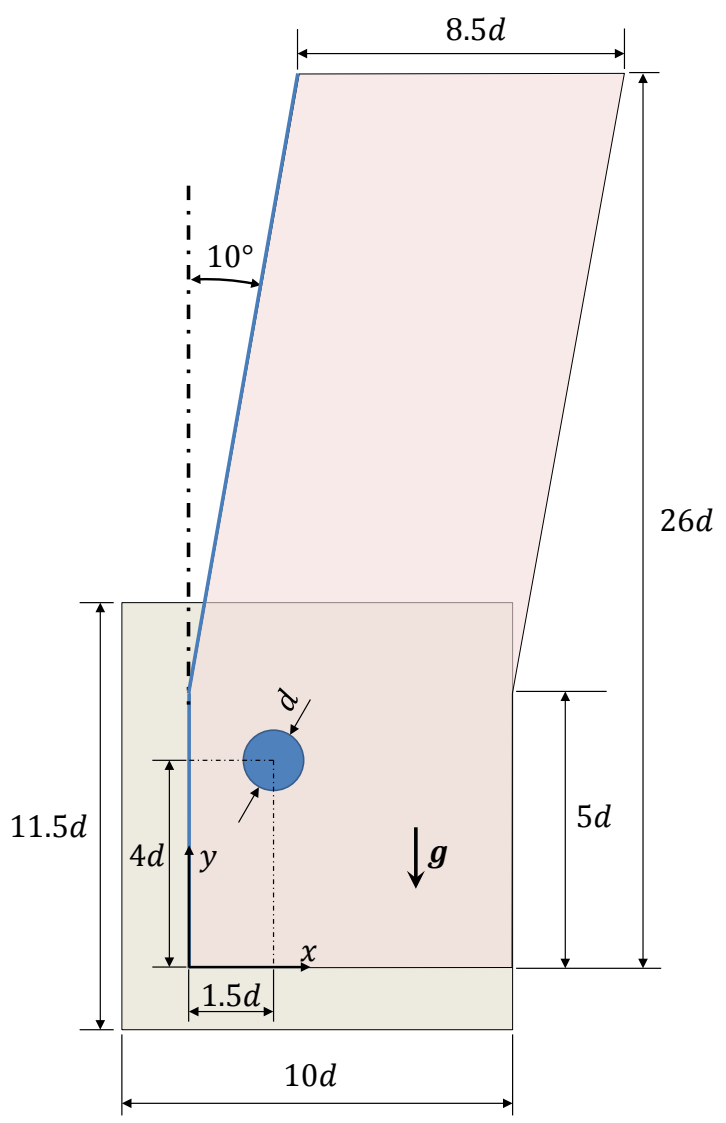

Figure 5: Outline of the initial set-up of the problem of a bubble bouncing through a highly-inclined plane. Simulation domains are highlighted in shaded boxes for both the complete domain case and the one with the moving mesh.

mesh case.

Two integral magnitudes are chosen to assess the aforementioned mesh resolutions. The first one is the average dimensionless velocity in the sliding state $\left\|\mathbf{v}_{\text {sliding }}^{*}\right\|=\left\|\mathbf{v}_{\text {sliding }}\right\| / \sqrt{d g}$, which is computed from the moment when the bubble starts interacting with the wall. This moment is determined as the point where the lateral velocity $\mathbf{v}_{\text {bubble, } x}$ ceases to be negligible compared to the vertical one $\mathbf{v}_{\text {bubble, } y}: \mathbf{v}_{\text {bubble, } x} / \mathbf{v}_{\text {bubble, } y}>$ 0.05, which happens at dimensionless time $t^{*}=t \sqrt{g / d}$ approximately equal to 1.8. Furthermore, the variation of the bubble shape is evaluated in terms of the circularity $\zeta_{2 D}$, which compares a perfect circular bubble against the actual one (see App. B for details on how to compute it). The value of the average circularity

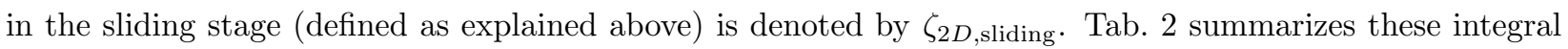
values for the different tested resolutions. It shows that the mesh convergence has been already achieved for meshes labelled as $M 2$. Therefore, a resolution of $h=d / 23$ seems enough to accurately reproduce the 
physics of the problem.

Furthermore, the proposed CLS+MM+IB method presents also a good performance concerning the mass conservation. By using this method, the obtained final mass error $E_{\mathrm{V}}$ for the meshes $M 1, M 2$ and $M 3$ is respectively $1.12 \cdot 10^{-11}, 6.64 \cdot 10^{-12}$ and $5.78 \cdot 10^{-12}$. Finally, the convergence rate has been computed by using the results of the sliding velocity shown in Tab. 2. Result of the finer mesh has been taken as reference value. Therefore, the obtained value of the convergence rate is 1.23 .

\begin{tabular}{ccc||cc||cc}
\hline Case & Mesh label & $h$ & $\mathbf{v}_{\text {sliding }}^{*}$ & $\zeta_{2 D, \text { sliding }}$ & $E_{\left\|\mathbf{v}_{\text {sliding }}^{*}\right\|}$ & $E_{\zeta_{2 D, \text { sliding }}}$ \\
\hline \multirow{3}{*}{ Full domain } & $M 1_{\mathrm{FD}}$ & $h=d / 15$ & 0.5413 & 0.8580 & $2.64 \%$ & $1.89 \%$ \\
& $M 2_{\mathrm{FD}}$ & $h=d / 23$ & 0.5519 & 0.8452 & $0.74 \%$ & $0.37 \%$ \\
& $M 3_{\mathrm{FD}}$ & $h=d / 30$ & 0.5560 & 0.8421 & - & - \\
\hline \multirow{3}{*}{ CLS+MM+IB method } & $M 1_{\mathrm{MM}}$ & $h=d / 15$ & 0.5488 & 0.8580 & $2.20 \%$ & $2.13 \%$ \\
& $M 2_{\mathrm{MM}}$ & $h=d / 23$ & 0.5398 & 0.8470 & $0.52 \%$ & $0.82 \%$ \\
& $M 3_{\mathrm{MM}}$ & $h=d / 30$ & 0.5370 & 0.8401 & - & - \\
\hline
\end{tabular}

Table 2: Integral results and mesh independence study for the problem of the passage of a bouncing bubble through a highly-inclined plane. Here $h$ is the characteristic cell size, $\left\|\mathbf{v}_{\text {sliding }}^{*}\right\|$ is the absolute value of the sliding velocity, $\zeta_{2 D \text {,sliding }}$ is average circularity in the sliding stage, $E_{\| \mathbf{v}_{\text {sliding }}^{*}} \|$ is the relative error of the absolute value of the sliding velocity referred to the case with denser mesh, and $E_{\zeta_{2 D} \text {,sliding }}$ is analogously the relative error of the average circularity in the sliding stage.

Further results are presented below, comparing those of $M 3$ meshes. First, the evolutions of the dimensionless velocity components $v_{y}^{*}=v_{\text {bubble, } y} / \sqrt{d g}$ and $v_{x}^{*}=v_{\text {bubble }, x} / \sqrt{d g}$ are presented in Fig. 6a and 6b. respectively. In these figures, it is clearly shown the oscillatory nature of the velocity evolution. The bubble speeds up from rest with almost null lateral velocity, until it starts interacting with the solid. In this bouncing stage, its velocity fluctuates around an average value remaining fairly constant with a very light increase. This oscillatory trend is also observed in the evolution of the dimensionless minimum distance from bubble centroid to the left wall $d_{\min }^{*}=d_{\min } / d$, which is plotted in Fig. 6c. This figure shows that the first stage of the bubble ascent is characterized by a slight increase in the distance to the wall. This is caused by the constriction effect of the left lateral wall, that tends to expel the bubble. After this period, this distance drops sharply. Finally, the evolution of the circularity $\zeta_{2 D}$ is plotted in Fig. 6d showing a clear fluctuating pattern around 0.85 approximately. All these plots show that the proposed CLS+MM+IB method provides reasonably good agreement in comparison with the reference data.

Finally, a comparison between profiles evolution is presented in Fig. 7. together with the bubble trajectory. This image highlights once more the oscillatory nature of the bubble path. During the bouncing stage, the bubble loses its symmetry, and it alternatively elongates towards right and left. Again, CLS+MM+IB method yields nearly indistinguishable results from the reference method. 


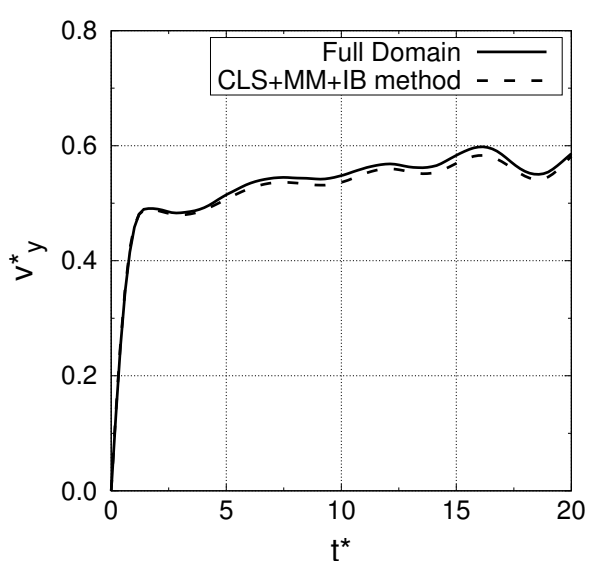

(a)

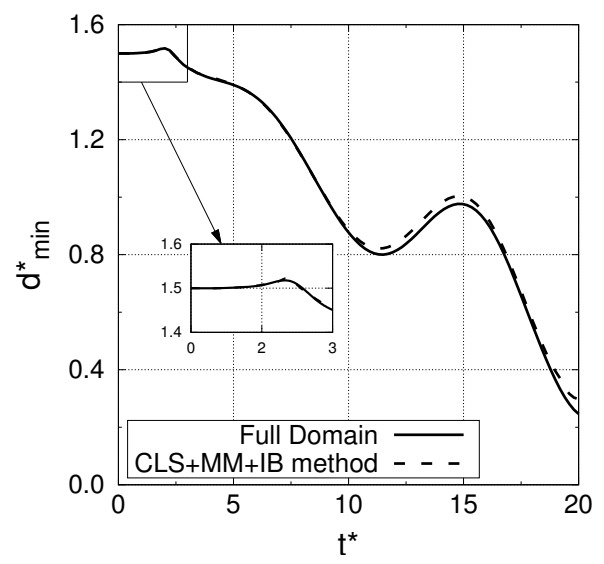

(c)

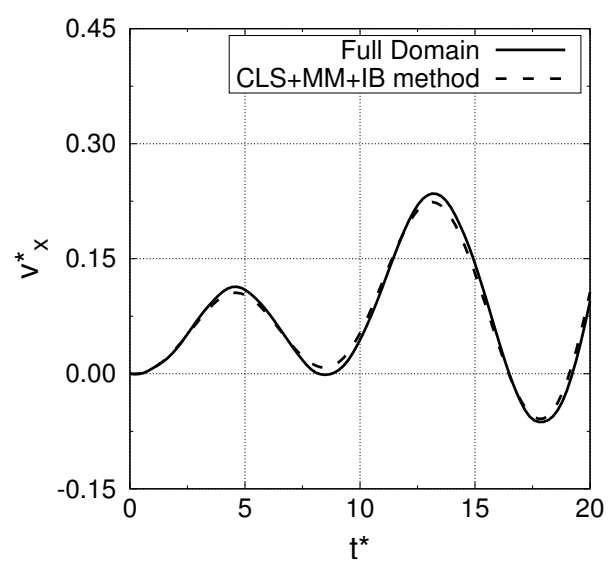

(b)

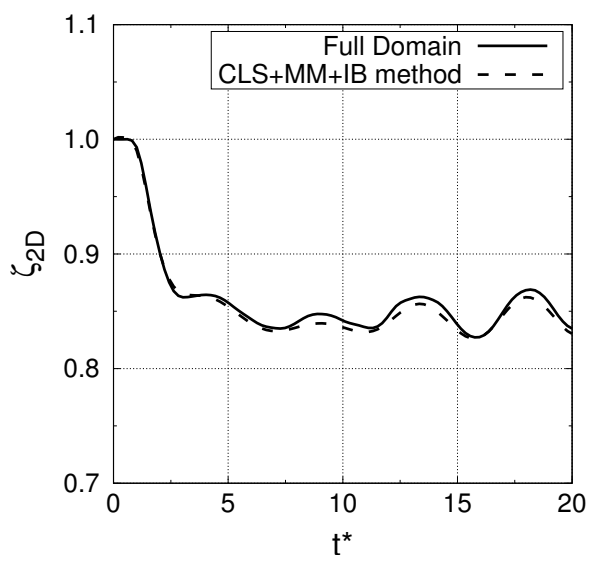

(d)

Figure 6: Result of the 2D problem of a bubble approaching an inclined plane. The graphs show (a) the vertical dimensionless velocity $v_{y}^{*}=v_{\text {bubble, } y} / \sqrt{d g}$, (b) the lateral dimensionless velocity $v_{x}^{*}=v_{\text {bubble }, x} / \sqrt{d g}$, (c) the dimensionless minimum distance $d_{\mathrm{min}}^{*}=d_{\mathrm{min}} / d$ from the bubble centroid to the wall, and (d) circularity $\zeta_{2 D}$. Those magnitudes are plotted against dimensionless time $t^{*}=t \sqrt{g / d}$, for the mesh resolution $h=d / 30$ (meshes $M 3_{\mathrm{FD}}$ and $M 3_{\mathrm{MM}}$ ).

The very small deviations between the results of both methods arise for three reasons. First, the mesh rotation makes the lateral distance from bubble centroid to the right boundary to vary slightly along the simulation, causing small disturbances in the velocity field. Second, the used open boundaries in the CLS+MM+IB method may also affect the far field. And third, the treatment of the IB and MM intrinsically introduce a small numerical error in the obtained results [36.

The great advantage of the proposed CLS+MM+IB method is the resources consumption savings. Tab. 3 summarizes some performance data to give an idea of those savings. In particular, simulation times are much 


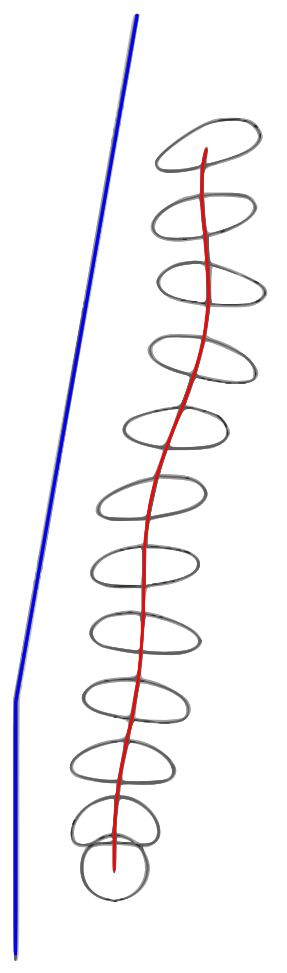

(a)

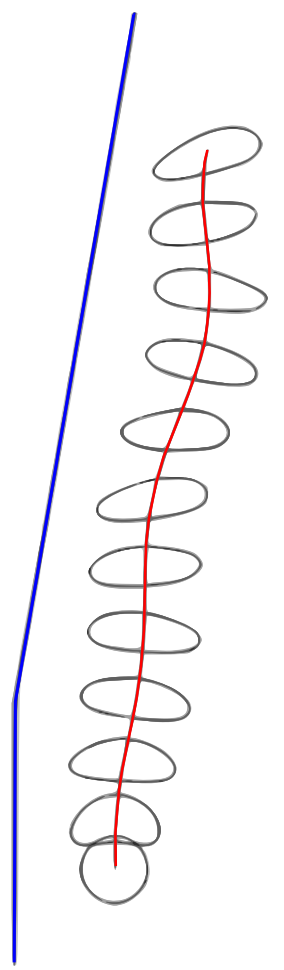

(b)

Figure 7: Profiles evolution in the problem of a bubble approaching a highly-inclined plane, for (a) the full domain case and (b) the CLS+MM+IB case. Successive profiles are plotted each 1.85 dimensionless time units.

lower compared with the case with a complete mesh (approximately one third). Furthermore, it is worth noting that the longer the simulation is, the more notable these differences are.

\subsection{Two-dimensional gravity-driven Taylor bubble through a curved channel}

In the present section, the $2 \mathrm{D}$ problem of an elongated bubble rising in a curved channel is addressed. The channel whereby the bubble evolves has a curved part connecting two straight sections: a vertical one and an inclined one. This case is particularly convenient to validate the rotating mesh process in constricting geometries (see Sec. 3.1). Therefore, the mesh does not exactly follow the motion of the bubble, but slides over the driving curve of the pipe. The results of the proposed CLS+MM+IB method are compared against a numerical simulation run using the complete domain, i.e. without immersed boundaries and moving mesh. As was stated in the section above, this way of comparing models provides a clear process to discern the deviations caused by the use of a moving mesh and an immersed boundary method.

Fig. 8 sketches the initial setting up of the numerical experiment, as well as the mesh dimensions for 


\begin{tabular}{ccc}
\hline Mesh label & $t_{\text {sim }, \mathrm{MM}} / t_{\text {sim }, \mathrm{FD}}$ & $N_{\mathrm{MM}} / N_{\mathrm{FD}}$ \\
\hline$M 1$ & 0.58 & 0.53 \\
$M 2$ & 0.55 & 0.49 \\
$M 3$ & 0.53 & 0.46 \\
\hline
\end{tabular}

Table 3: Resources consumption comparison in the problem of a bubble approaching a highly-inclined plane. Here, the results of the proposed CLS+MM+IB method are compared against those obtained by using a standard approach with the complete domain. $t_{\mathrm{sim}, \mathrm{MM}} / t_{\mathrm{sim}, \mathrm{FD}}$ represents the ratio between simulation times and $N_{\mathrm{MM}} / N_{\mathrm{FD}}$ the ratio between total mesh sizes.

both cases. As shown in this figure, the initial shape of the bubble is a two-dimensional cylinder with two hemispheres. The diameter $d$ of the initial bubble is equal to $0.8 D$, where $D$ is the diameter of the pipe. The length of the initial bubble is $1.1534 D$, giving rise to an equivalent total volume equal to $\pi D^{2} / 4$. The mesh width in the proposed CLS+MM+IB method is equal to the diameter of the pipe $D$ plus a value $e$ given by:

$$
e=\left(R_{\Upsilon}-\frac{d}{2}\right)-\sqrt{\left(R_{\Upsilon}-\frac{d}{2}\right)^{2}-h_{i}^{2}} \approx 0.292 D
$$

where $R_{\Upsilon}=4.5 D$ is the turning radius of the driving curve, and $h_{i}=1.5 D$ is the chosen distance from the bubble centroid to the inlet. This assures that the mesh is able to cover the whole transversal section of the pipe during its ascent. Eq. 24 is a particular expression for circular turns, and it should be expressly revised in any other case. See Sec. 3.1 for further notes on the calculus of this distance. Additionally, distances from bubble centroid to inlet and outlet are set based on the indications given in [35.

Different mesh resolutions are tested in both the case with the complete domain, and the case with moving mesh. Thus, three different meshes are used in each case, namely from $M 1$ to $M 3$ in order of increasing resolution. These meshes are unstructured and composed of triangular control volumes. The chosen resolutions are $D / 50, D / 75, D / 100$.

For this problem, dimensionless numbers characterizing the flow regime are defined as follows:

$$
\eta_{\rho}=\frac{\rho_{1}}{\rho_{2}} \quad, \quad \eta_{\mu}=\frac{\mu_{1}}{\mu_{2}} \quad, \quad E o=\frac{g D^{2} \rho_{1}}{\sigma} \quad, \quad M o=\frac{g \mu_{1}^{4}}{\rho_{1} \sigma^{3}}
$$

Here $\eta_{\rho}=756.02, \eta_{\mu}=4597.70, E o=100.0$ and $M o=0.015$. Note that the definition of those dimensionless numbers differs from the one given in Eq. 23 for the previous problem. The chosen slug flow regime has been broadly studied, starting from the original work by Bugg and Sad [66].

Some integral magnitudes are shown in Tab. 4 in order to assert the adequateness of the different meshes tested. Two integral values are shown in that table. On the one hand, the dimensionless sliding velocity $\left\|\mathbf{v}_{\text {sliding }}^{*}\right\|=\left\|\mathbf{v}_{\text {sliding }}\right\| / \sqrt{D g}$ is presented for the different mesh resolutions. This value is obtained by averaging the dimensionless bubble velocity during the inclined ascent, i.e from dimensionless time $t^{*}=t \sqrt{g / D}$ 


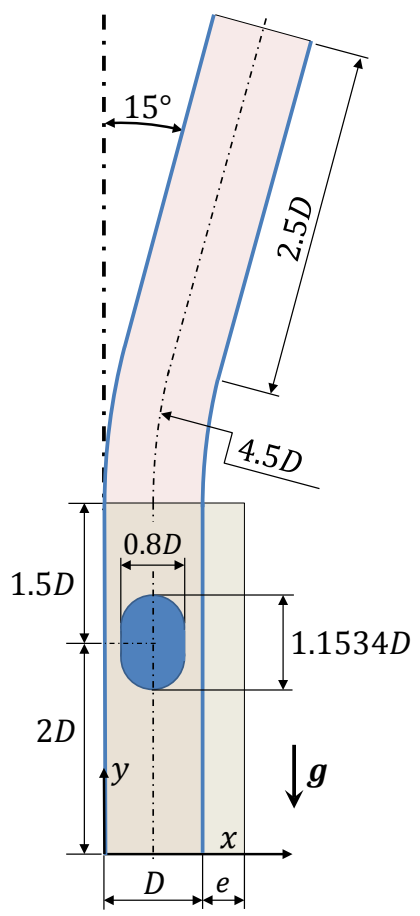

Figure 8: Outline of the initial set-up of the problem of a Taylor bubble evolving in a curved channel. The initial length of the bubble is set to $1.1534 D$ in order to get a $2 \mathrm{D}$ volume of the bubble equal to $\pi D^{2} / 4$. Simulation domains are highlighted in shaded boxes for both the complete domain case and the one with the moving mesh. The mesh width in the moving mesh case is equal to $D+e$, where $e \approx 0.292 D$.

approximately equal to 12.6. On the other hand, the shape of the bubble is evaluated in terms of the deformation parameter $\Delta=(L-B) /(L+B)$ in the sloping part of the ascent, where $L$ is the length of the bubble from nose to tail, and $B$ is its average transversal length. The results comparison shows that $h=D / 75$ seems a reasonable resolution to accurate reproduce the ascent of the Taylor bubble.

By using the CLS+MM+IB method, the mass error $E_{\mathrm{V}}$ is kept under control. For the meshes $M 1, M 2$ and $M 3$, the obtained values of this magnitude at the end of the simulation are respectively $8.72 \cdot 10^{-13}$, $2.78 \cdot 10^{-13}$ and $1.33 \cdot 10^{-13}$.

Additional results of the current problem are shown in Fig.9. for $M 3$ meshes. First, the vertical component of the dimensionless velocity $v_{y}^{*}=v_{\text {bubble }, y} / \sqrt{D g}$ is plotted against dimensionless time $t^{*}=t \sqrt{g / D}$. This magnitude rises from zero to a terminal value, which is slightly modified due to the change of the channel inclination. On the other hand, the evolution of the lateral dimensionless velocity $v_{x}^{*}=v_{\text {bubble }, x} / \sqrt{D g}$ shows 


\begin{tabular}{ccccccc}
\hline Case & Mesh label & $h$ & $\left|\mathbf{v}_{\text {sliding }}\right|$ & $\Delta$ & $E_{\left|\mathbf{v}_{\text {sliding }}\right|}$ & $E_{\Delta}$ \\
\hline \multirow{3}{*}{ Full domain } & $M 1_{\mathrm{FD}}$ & $h=D / 50$ & 0.2104 & 0.2646 & $0.32 \%$ & $4.74 \%$ \\
& $M 2_{\mathrm{FD}}$ & $h=D / 75$ & 0.2109 & 0.2722 & $0.10 \%$ & $2.02 \%$ \\
& $M 3_{\mathrm{FD}}$ & $h=D / 100$ & 0.2111 & 0.2778 & - & - \\
\hline \multirow{3}{*}{ CLS+MM+IB method } & $M 1_{\mathrm{MM}}$ & $h=D / 50$ & 0.2014 & 0.2528 & $1.93 \%$ & $5.72 \%$ \\
& $M 2_{\mathrm{MM}}$ & $h=D / 75$ & 0.2025 & 0.2590 & $1.41 \%$ & $3.39 \%$ \\
& $M 3_{\mathrm{MM}}$ & $h=D / 100$ & 0.2053 & 0.2682 & - & - \\
\hline
\end{tabular}

Table 4: Integral results and mesh independence study of the problem of a Taylor bubble rising in a pipe with a change in its inclination. Here $h$ is the characteristic cell size, $\left|\mathbf{v}_{\text {sliding }}\right|$ is the absolute value of the sliding velocity (in the inclined section), $\Delta$ is the deformation parameter at the sloping part, $E_{\left|\mathbf{v}_{\text {sliding }}\right|}$ is the relative error of the absolute value of the sliding velocity referred to the case with denser mesh, and $E_{\Delta}$ is analogously the relative error of the deformation parameter at the inclined section.

a similar behaviour. It presents two clearly distinct sections: one before the pipe elbow, and another one after the pipe elbow. In the first one, this velocity component is close to zero. In the passage of the bubble through the elbow, an adaptation region is identified. Here, $v_{x}^{*}=v_{\text {bubble, } x} / \sqrt{D g}$ rises and eventually stabilizes in the inclined zone. Both methods provide very close results.

Finally, Fig. 10 sketches the profiles evolution for both methods. In the corresponding image of the proposed CLS+MM+IB method, the positions of the mesh at each time instant are also represented. The figure shows the well-defined rotation process of the mesh, following the driving curve of the channel. All of these results suggest that the CLS+MM+IB method is certainly capable of reproducing the physics of the problem with enough level of accuracy. Small differences are explained in terms of the presence of open boundaries and numerical issues (e.g. the meshes, although sharing the same resolution, are not identical).

In a reference to the resource consumption comparison, the proposed CLS+MM+IB method performs much better than a traditional DNS approach with a complete domain. Tab. 5 presents a comparison among simulation times and mesh sizes. Note that if the physical domain were bigger, those savings would be much more notable.

\subsection{Three-dimensional gravity-driven buoyant drop in a constricted circular channel}

In the present section, we analyze the motion and evolution of a drop as it passes through a periodically constricted pipe. The proposed CLS+MM+IB method is enforced to tackle this problem in a full three-dimensional domain. Some of the advantages of the proposed method are clearly highlighted in this problem. First, a three-dimensional approach is only computationally affordable by using a domain optimization method, i.e. the moving mesh technique in this case. Any method involving the complete 3D domain would be prohibitive in terms of the DNS, as the physical domain needs to be very long to collect average data in the periodic motion of the drop. Additionally, the use of an immersed boundary method allows a 


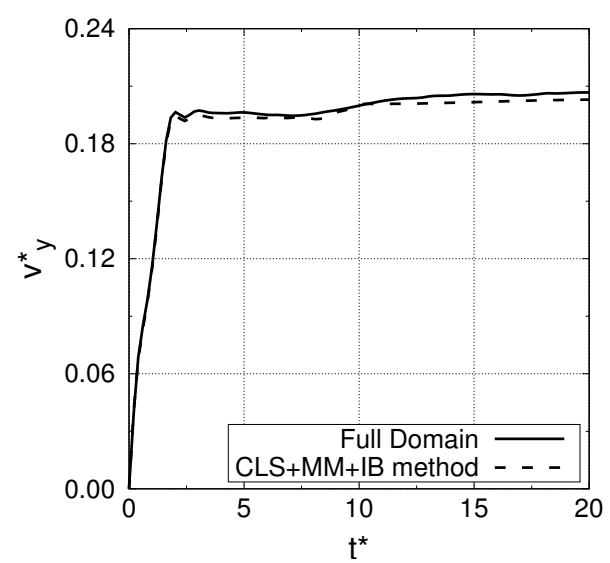

(a)

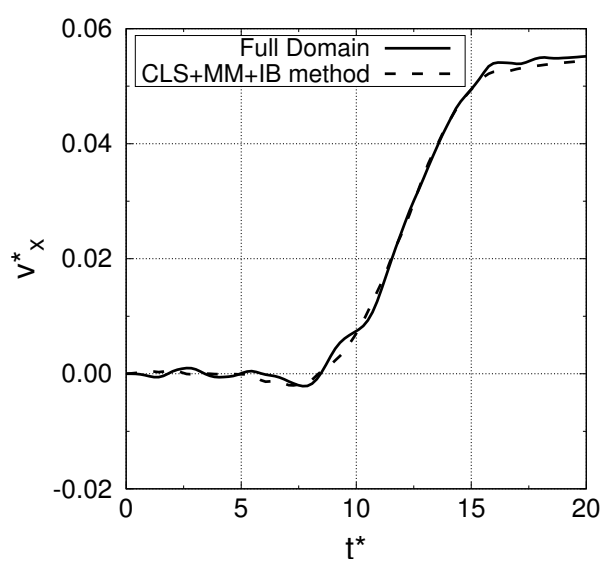

(b)

Figure 9: Result of the 2D problem of a Taylor bubble turning in a curved pipe. The graphs show (a) the vertical dimensionless velocity $v_{y}^{*}=v_{\text {bubble }, y} / \sqrt{D g}$, and (b) the lateral dimensionless velocity $v_{x}^{*}=v_{\text {bubble }, x} / \sqrt{D g}$. Those magnitudes are plotted against dimensionless time $t^{*}=t \sqrt{g / D}$, for the mesh resolution $h=d / 100$ (meshes $M 3_{\mathrm{FD}}$ and $\left.M 3_{\mathrm{MM}}\right)$.

simple mesh generation process, as well as facilitates parametric studies or geometrical modifications (e.g. in the wavelength or amplitude of the constrictions). A classical approach would have required a complete re-meshing process when varying the original conditions, while the present methodology would just need a redefinition of the surface mesh (STL) of the solid, with no changes in the fluid mesh.

Numerical data of Olgac et al. [17] is taken as reference, which in turn is based on the experimental work of Hemmat and Borhan [4]. On the basis of these references, the set-up sketched in Fig. 11 is used in the present study. The pipe is trivially generated by radial extrusion of a sinusoidal curve. Therefore, the wavelength $\lambda$ is set to $4 D$, where $D$ is the average pipe diameter, and the amplitude $A$ is set to $0.07 D$. The drop initial axial position is chosen to be at the point of maximum tube diameter $D+2 A$. A drop dimensionless diameter is defined as $k=d / D$, where $d$ is its dimensional diameter. In this experiment, $k$ is set to 0.78 . Distances from drop centroid to inlet $(1.8 D)$ and outlet $(2.2 D)$ are chosen considering the notes given in [35]. Those distances assure that the presence of the open boundaries does not disturb the motion of the drop.

The simulation domain is cylindrical, with a diameter equal to $D+2 A$. This size guarantees that the mesh covers the whole transversal section of the solid throughout the ascent. This domain has been meshed using unstructured triangular prisms. The meshes have been generated by a constant step extrusion of a two-dimensional grid along the cylinder axis, being $h$ the step size, and also the characteristic size of the triangular elements of the extruded grid. Three different meshes have been considered, labelled from $M 1$ 


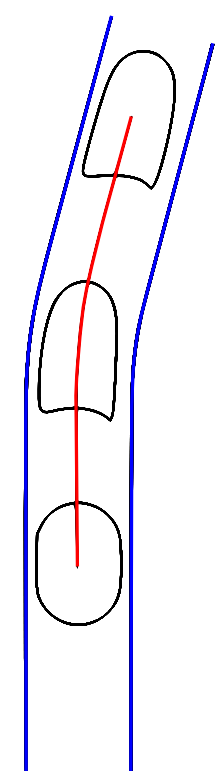

(a)

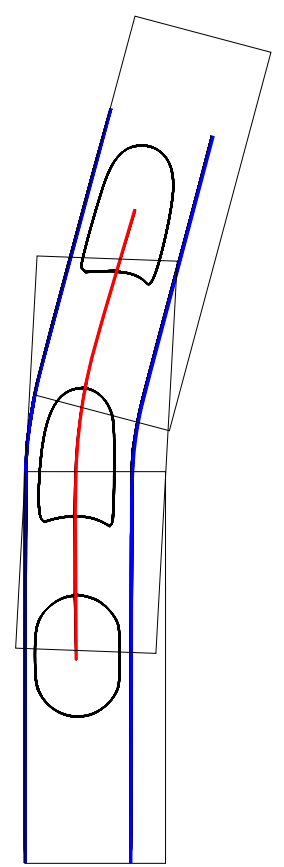

(b)

Figure 10: Profiles evolution in the problem of a Taylor bubble turning in a curved pipe. Results correspond to (a) the full domain case and (b) the CLS+MM+IB one. Successive profiles are plotted at 0, 11.9 and 20 dimensionless time units.

to $M 3$ in increasing order of mesh resolution $h$. This resolution has been set by dividing the pipe average diameter $D$ into 22, 30 and 39, respectively. See Tab. 6 for a complete description of these meshes. The simulation domain is moved following the ascent of the drop as explained in Sec. 3.1. Nevertheless, for this particular case the procedure explained in that section for constricting geometries collapses in a linear translation, due to the intrinsic axisymmetric of the problem.

The flow regime is characterized by the dimensionless numbers given in Eq. 25. Therefore, $\eta_{\rho}=1.200$, $\eta_{\mu}=0.7565, E o=270.9$ and $M o=6.539$. These flow conditions are obtained when using a two-phase system composed by Diethylene glycol-glycerol (63.8wt\%) as suspending fluid and UCON - 285 as drop fluid [17]. It corresponds to the system labeled as DEGG12 of the references [17, 4].

Results are expressed in terms of dimensionless quantities. Time and velocities are made dimensionless using the following reference values, respectively:

$$
t_{\mathrm{ref}}=\frac{2 \mu_{1}}{\left(\rho_{1}-\rho_{2}\right) g D} \quad ; \quad \mathrm{v}_{\mathrm{ref}}=\frac{\left(\rho_{1}-\rho_{2}\right) g D^{2}}{4 \mu_{1}}
$$




\begin{tabular}{ccc}
\hline Mesh label & $t_{\text {sim }, \mathrm{MM}} / t_{\text {sim }, \mathrm{FD}}$ & $N_{\mathrm{MM}} / N_{\mathrm{FD}}$ \\
\hline$M 1$ & 0.67 & 0.65 \\
$M 2$ & 0.65 & 0.61 \\
$M 3$ & 0.59 & 0.56 \\
\hline
\end{tabular}

Table 5: Resources consumption comparison in the problem of a Taylor bubble turning in a curved pipe. Results of the proposed CLS+MM+IB method are compared against those obtained by using a standard DNS approach with the complete domain. $t_{\mathrm{sim}, \mathrm{MM}} / t_{\mathrm{sim}, \mathrm{FD}}$ represents the ratio between simulation times and $N_{\mathrm{MM}} / N_{\mathrm{FD}}$ the ratio between total mesh sizes.

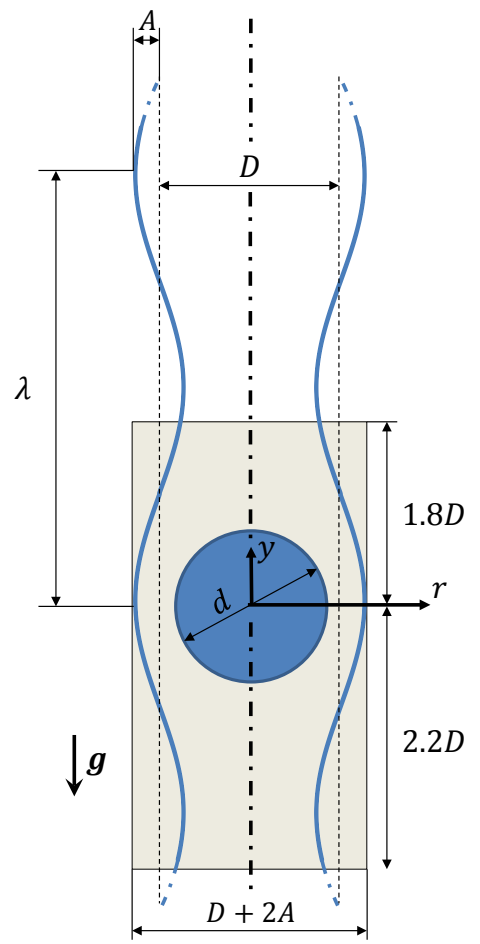

Figure 11: Initial set-up of the problem of a drop rising in a periodically constricted circular channel. Here, $D$ is the average pipe diameter, $d$ is the initial drop diameter, $\lambda$ is the wavelength of the corrugations and $A$ is their amplitude. The shaded grey box represents the simulation domain.

For distances, $\lambda=4 D$ is taken as reference magnitude.

The average dimensionless terminal velocity of the drop $\left|\overline{\mathbf{v}}_{\text {drop }}^{*}\right|$ has been taken as reference value to measure the accurateness of a specific simulation. Tab. 7 shows the value of this magnitude for the different 


\begin{tabular}{ccccc}
\hline Mesh label & Mesh size & $\begin{array}{c}\text { Cells per } \\
\text { plane }\end{array}$ & $N_{\text {planes }}$ & $h$ \\
\hline$M 1$ & $1.1 \cdot 10^{5}$ & 1290 & 84 & $D / 22$ \\
$M 2$ & $3.9 \cdot 10^{5}$ & 2744 & 132 & $D / 33$ \\
$M 3$ & $5.9 \cdot 10^{5}$ & 3872 & 156 & $D / 39$ \\
\hline
\end{tabular}

Table 6: Description of the meshes used in the problem of a drop passing through a periodically constricted pipe, where $N_{\text {planes }}$ is the number of planes in which the vertical axis is divided.

meshes tested, as well as a comparison against reference data. Additionally, mass error $E_{\mathrm{V}}$ is kept under control for the three tested meshes $(M 1, M 2$ and $M 3)$. The obtained values for this magnitude at the end of the simulation are respectively $6.83 \cdot 10^{-8}, 2.44 \cdot 10^{-8}$ and $4.74 \cdot 10^{-9}$.

\begin{tabular}{cc|c|c}
\hline Results & $\overline{\mathbf{v}}_{\text {drop }}^{*}$ & $E_{\left|\overline{\mathbf{v}}_{\text {drop }}^{*}\right|}$ \\
\hline Present work $M 1$ & $6.686 \cdot 10^{-3}$ & $26.07 \%$ \\
Present work $M 2$ & $7.832 \cdot 10^{-3}$ & $13.40 \%$ \\
Present work $M 3$ & $8.558 \cdot 10^{-3}$ & $5.37 \%$ \\
Olgac et al. [17] & $9.648 \cdot 10^{-3}$ & $6.68 \%$ \\
Hemmat and Borhan [4] & $9.044 \cdot 10^{-3}$ & - \\
\hline
\end{tabular}

Table 7: Integral results of the problem of a drop rising through a periodically constricted channel, in comparison with reference data [17, 4]. $\left|\overline{\mathbf{v}}_{\text {drop }}^{*}\right|$ is the average dimensionless terminal velocity of the drop, and $E_{\left|\overline{\mathbf{v}}_{\text {drop }}^{*}\right|} \mid$ is the relative error associated with that magnitude, in comparison with the reported results of Hemmat and Borhan 4 .

Further outcomes of the current study are presented in subsequent figures. First, a comparison among profile shapes are presented in Fig. 12 In this figure, results form the present work are compared with those by Olgac et al. 17] and Hemmat and Borhan [4. Those profiles are plotted when the drop is in its periodical steady state, and when it is passing through the minimum transversal section of the pipe (throat) and the maximum one (expansion). The snapshots are qualitatively in good agreement with the reference data. Slight disagreements are explained based on the ambiguity of locating the drop at a specific axial position, as the profile shape notably changes in a very tight range.

Time evolutions of the dimensionless velocity $\mathbf{v}_{\text {drop }}^{*}$ and risen distance $y^{*}$ are depicted in Fig. 13 for the different tested meshes. A well-defined oscillatory behaviour is observed in the rising steady state. The drop speeds up during the expansion passage, and slows down while approaching the throat. The mesh convergence behaviour is clearly seen in these graphs. Additionally, Fig. 14 sketches some geometrical

\footnotetext{
${ }^{1}$ Right column of Fig. 12 was reprinted from Chemical Engineering Communications, 148-150, M. Hemmat and A. Borhan, Buoyancy-driven motion of drops and bubbles in periodically constricted capillary, p. 371, Copyright 1996, with permission from Taylor \& Francis.
} 


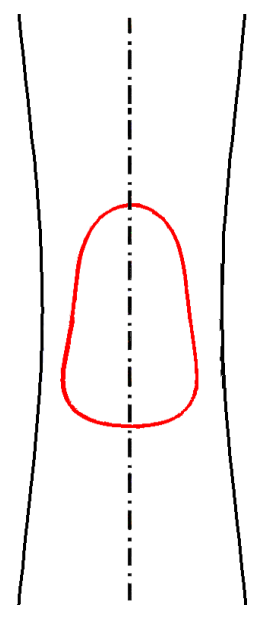

(a)

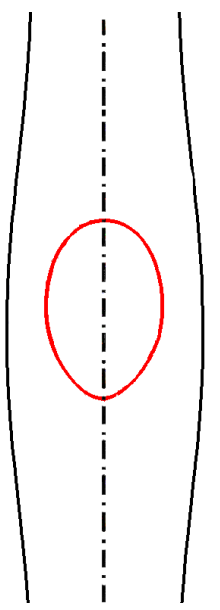

(d)

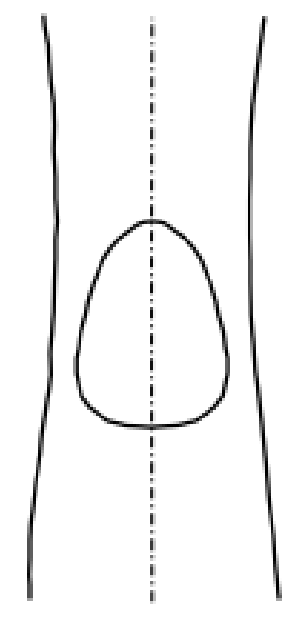

(b)

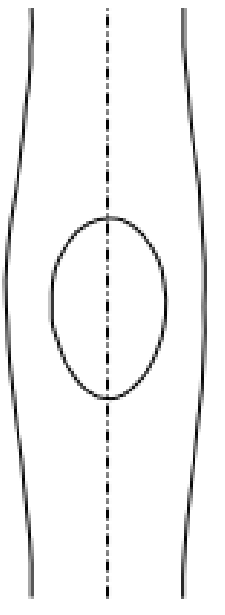

(e)

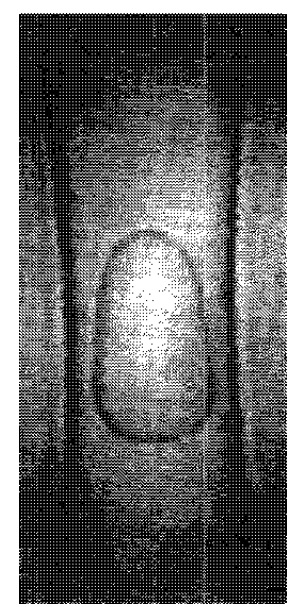

(c)

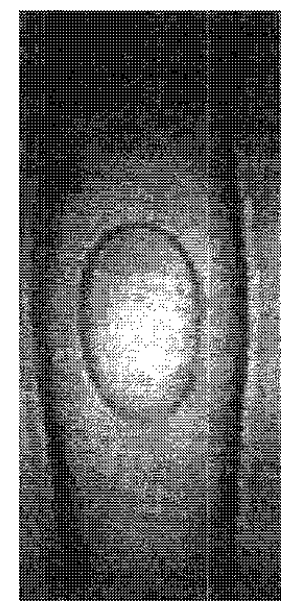

(f)

Figure 12: Comparison of the profile shapes among different results. Graphs (a) and (d) correspond to the present work, (b) and (e) to Olgac et al. [17, and (c) and (f) to Hemmat and Borhan 4] 1]. The snapshots of the first row correspond to the moment when the drop is passing through the channel throat, and those of the second row correspond to the moment when the drop is located at the expansion section.

magnitudes throughout a single period of corrugation, in comparison with reference data of 4 . Note that there is a discrepancy in the reference for the dimensionless drop diameter $k$. In [4 this parameter is set to $k=0.73$, and the chosen one in the present work is $k=0.78$. The dimensionless distance $\hat{y}^{*}$ indicates the axial position of the advancing drop meniscus, varying from 0 to 1 (from a specific throat to the next). In Fig. 14 the deformation parameter $\Delta$ determines the ratio of the perimeter of the deformed drop profile to that of the equivalent spherical drop. Additionally, the dimensionless drop length $L^{*}$ is defined as the 
maximum axial drop distance. Those magnitudes were found to be periodic in the steady ascent of the drop. Despite the aforementioned discrepancy in the dimensionless drop diameter $k$, results are in reasonable agreement with those of [4, and overall trends are very well represented.

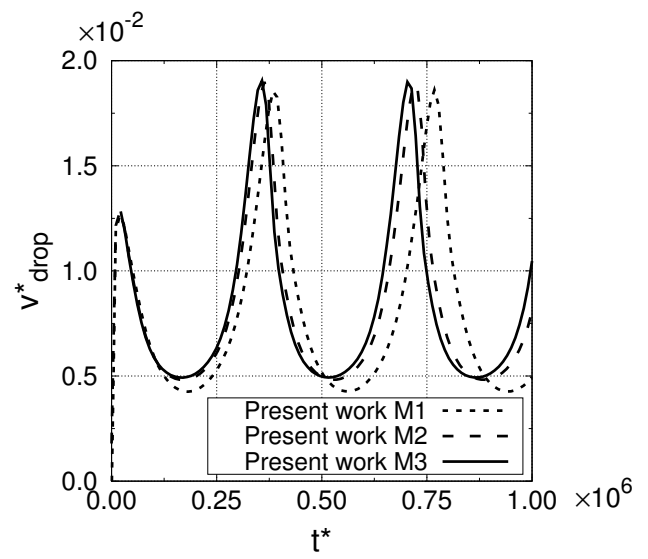

(a)

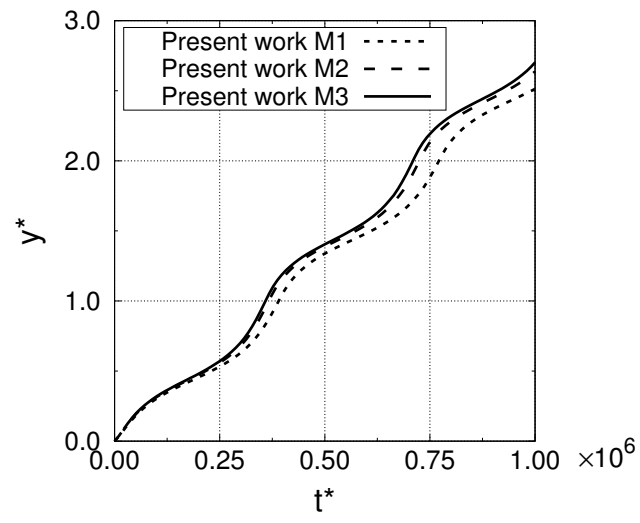

(b)

Figure 13: Evolution of (a) dimensionless drop velocity and (b) dimensionless axial position of the centroid of the drop, against dimensionless time $t^{*}$, for the three different meshes tested in the present work.

Furthermore, Fig. 15 depicts the streamlines and the vorticity field in a plane containing the $y$-axis of the corrugated channel. In that figure, four states of the periodic steady motion of the drop are presented: the drop passing through the throat, passing through the expansion, and the upward and downward intermediate states. When the drop is at the throat, it presents an elongated mushroom shape (Fig. 15a) with a vortex at the rear end of the drop, close to the point of minimum distance between the drop interface and the wall. As the drop rises and expands, the drop tail becomes narrower, acquiring a bullet shape (Fig. 15b). Likewise, the vortex shifts towards the nose of the rising drop. This vortex reaches maximum intensity in the expansion (Fig. 15c), where the drop shape becomes elliptical. Finally, at the early stage of the constriction (Fig. 15d), the drop tail begins to widen, and the vortex recovers its initial position at the rear end of the drop.

\subsection{Three-dimensional gravity-driven unconstrained drop impinging on a horizontal plane}

The last problem considered to validate the proposed CLS+MM+IB method is the 3D approach of a drop against a horizontal rigid plane. The study of this problem is relevant in fields as mineral flotation, sprays or cooling of nuclear reactors. It constitutes a very interesting problem to solve with the proposed methodology, as some of the main advantages discussed in Sec. 4 are plainly shown here. The experimental work by Klaseboer et al. [8] is taken as reference. In this paper, twelve flow conditions are tested, studying the trajectory of the drop when approaching the plane at its terminal velocity. We chose one of those experiments, and we simulated it by using the outlined CLS+MM+IB methodology. 


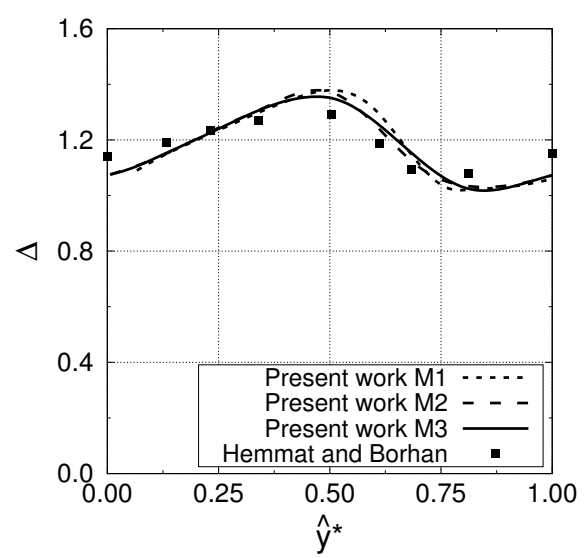

(a)

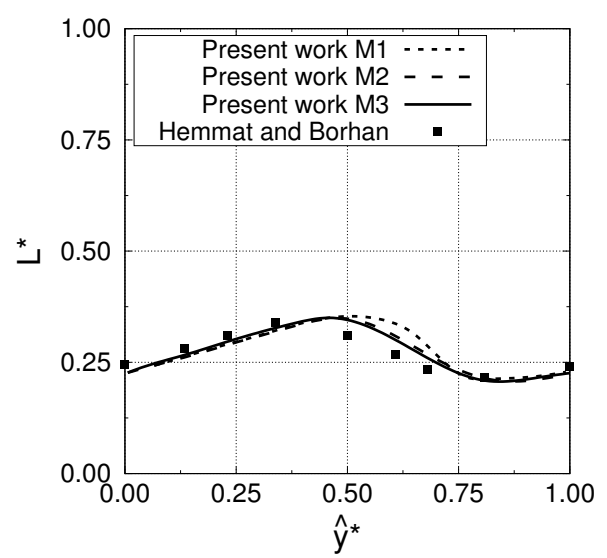

(b)

Figure 14: Evolution of (a) deformation parameter $\Delta$, and (b) dimensionless length $L^{*}$ throughout a single period of corrugation for the different mesh resolutions tested in the present work. Results are compared against those by Hemmat and Borhan [4]. The position of the drop is controlled by the dimensionless distance $\hat{y}^{*}$, which indicates the axial position of the advancing drop meniscus.

By using the proposed method, only a small computational domain around the drop is taken. Initially, the drop is at rest, and the horizontal solid is placed far away from the drop, outside of the simulation domain. When the drop achieves its steady state, the solid is suddenly moved at the upper limit of the computational domain. This procedure constitutes a major improvement in comparison with other DNS approaches to this problem. With the proposed CLS+MM+IB method, the distance which the drop travels before impinging on the wall is unimportant, as it can be dynamically set during the simulation. This feature facilitates the carrying out of parametric studies, and the easy modification of the flow conditions (e.g. the inclination of the solid or its geometry).

The initial arrangement of the problem is sketched in Fig. 16. Initially, the drop is spherical-shaped with a diameter equal to $d$. The initial distance from the drop to the plane $y_{\text {plane }}$ is set to a high value (e.g. $\left.y_{\text {plane }}=100 d\right)$. The plane is suddenly moved from that position to the upper limit of the domain at the moment when the drop achieves its terminal state. The motion of the drop within the domain is unbounded until the interaction with the plane starts. In order to assure this condition, distances form the bubble to the boundaries are chosen based on the notes given in [35, 67]. By doing this, we aim to find a compromise between domain size and disturbance of the solution.

Flow regime has been set by choosing the following dimensionless numbers (see Eq. 23): $\eta_{\rho}=1.1547$, $\eta_{\mu}=1.6949, E_{o}=0.1502$ and $M o=3.066 \cdot 10^{-11}$. This flow regime corresponds to the system labeled as 1 in the work of Klaseboer et al. [8]. 


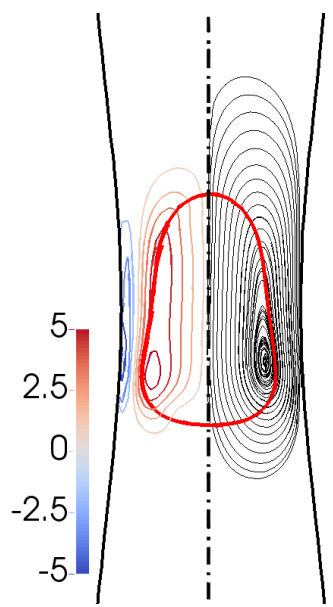

(a)

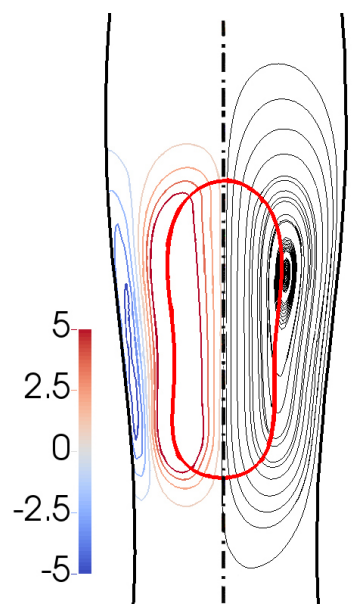

(b)

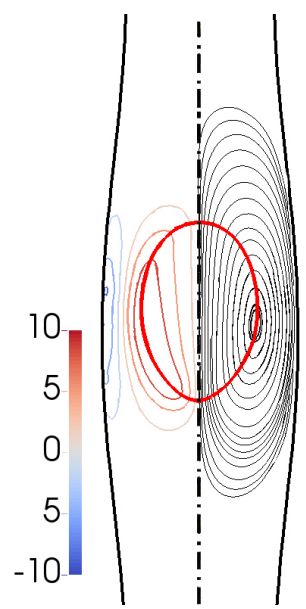

(c)

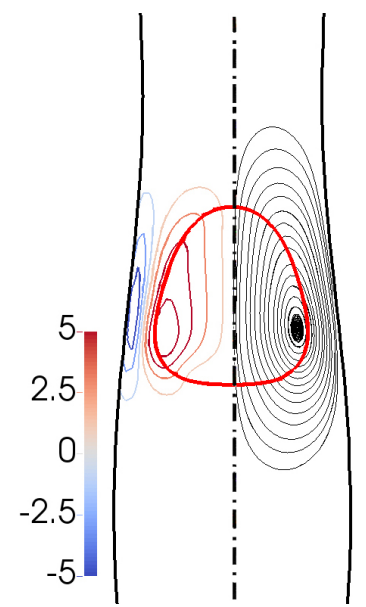

(d)

Figure 15: Streamlines and vorticity field $\left(s^{-1}\right)$ in a plane containing the $y$-axis of the channel. Different instants are obtained through the periodic steady motion of the drop, at (a) the throat, (c) the expansion, and (b) and (d) the upward and downward intermediate points.

The employed meshes are fully unstructured and composed by tetrahedral control volumes. The location of the drop will remain steady within the simulation domain, as the mesh is moved following its ascent. This behaviour can be exploited during the mesh design process. Therefore, the used meshes have a dense core of a diameter equal to $d$ around the initial position of the drop, with a characteristic cell size of $h_{\text {min }}$ A radial exponential growth is imposed over the cell sizes, reaching the maximum value $h_{\text {max }}$ at the borders (see Fig. 16b). Tab. 8 summarizes the meshes employed in the present study. Those meshes are obtained by varying $h_{\min }$ and $h_{\max }$ sizes.

\begin{tabular}{cccc}
\hline Mesh label & Mesh size & $h_{\min }$ & $h_{\max }$ \\
\hline$M 1$ & $5.5 \cdot 10^{5}$ & $d / 25$ & $d / 3$ \\
$M 2$ & $9.0 \cdot 10^{5}$ & $d / 35$ & $d / 4$ \\
$M 3$ & $1.8 \cdot 10^{6}$ & $d / 45$ & $d / 5$ \\
\hline
\end{tabular}

Table 8: Description of the meshes used in the problem of a drop impinging on a horizontal plane, where $h_{\text {min }}$ and $h_{\max }$ are the minimum and the maximum cell sizes, respectively.

Velocities are made dimensionless by means of the reference value $\sqrt{d g}$. For the time, the reference magnitude is $\sqrt{g / d}$. Finally, distances are made dimensionless by using the initial drop diamiter $d$ as reference value.

The dimensionless terminal distance $d_{\text {final }}^{*}$ from drop centroid to the wall at the final steady point has 


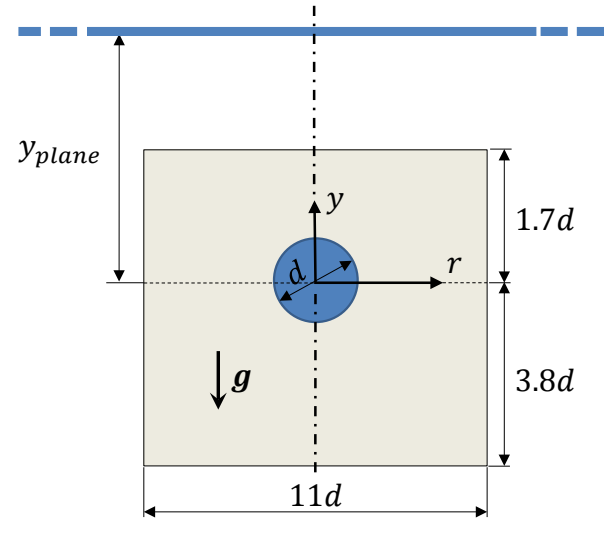

(a)

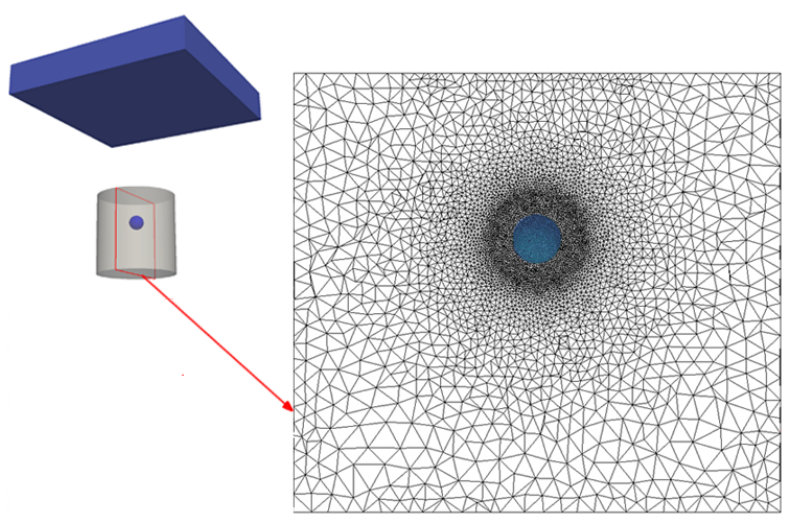

(b)

Figure 16: Initial set-up of the problem of a drop impinging on an immersed horizontal wall. The graphs show (a) the initial arrangement and (b) the mesh configuration. $d$ is the drop initial diameter and $y_{\text {plane }}$ is the initial distance from drop centroid to the solid plane.

been taken as the reference integral result to assert the mesh independence. Tab. 9 shows a comparison of the obtained results.

\begin{tabular}{ccc}
\hline Results & $d_{\text {final }}^{*}$ & $E_{d_{\text {final }}^{*}}$ \\
\hline Present work $M 1$ & 0.4645 & $4.05 \%$ \\
Present work $M 2$ & 0.4694 & $3.02 \%$ \\
Present work $M 3$ & 0.4841 & - \\
\hline
\end{tabular}

Table 9: Integral results for the problem of a drop rising and impacting against a solid horizontal plane. The magnitude $d_{\text {final }}^{*}$ represents the dimensionless terminal distance from drop centroid to the wall, and $E_{d_{\text {final }}^{*}}$ is the relative error associated with this distance, in comparison with the results obtained by using the finer mesh.

Further results are presented in subsequent figures. Fig. 17 shows the time evolution of some magnitudes of the problem. First, the dimensionless distance $d^{*}$ from the drop centroid to the plate has been represented against dimensionless time $t^{*}$ (Fig. 17a). Results include those obtained with the three tested meshes, as well as those of Klaseboer et al. 8. Present results are in good agreement with the reference data. When studying the impact of drops/bubbles against obstacles, the mass conservation error becomes a paramount concern. This is because the immersed boundary method should work properly when acting together with the chosen interface tracking technique. To evaluate this issue, Fig. $17 \mathrm{~b}$ represents the evolution of the mass error $E_{V}$, defined as the relative error of the volume of the drop $V_{2}$ in comparison with the initial one (see 
App. B. This error remains on the order of $10^{-8}$ throughout the simulation (which is a considerable small value).

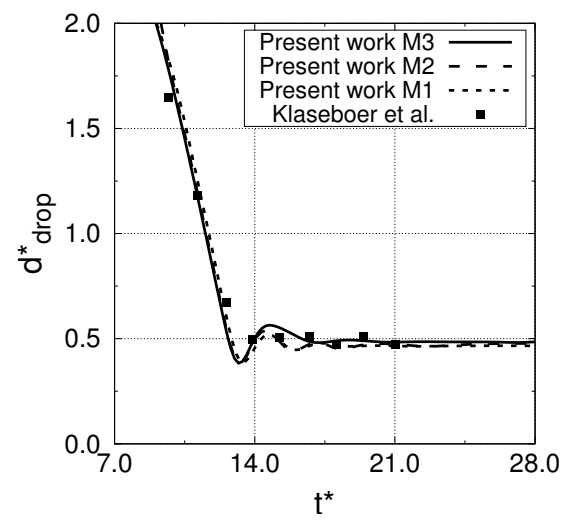

(a)

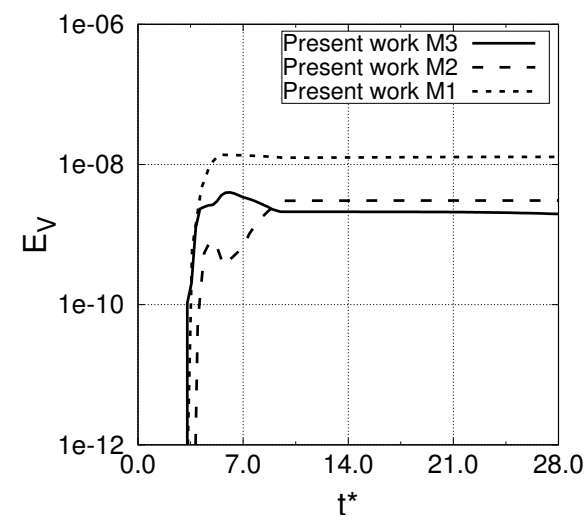

(b)

Figure 17: Evolution of (a) dimensionless distance from the drop centroid to the plate and (b) mass error, against dimensionless time $t^{*}$, for the three different meshes tested in the present work. Results are compared against those of Klaseboer et al. [8].

Finally, some snapshots of the impinging process have been plotted in Fig. 18 . The velocity and the pressure fields are represented over successive time instants from the moment when the drop achieves its terminal velocity. In the rising state, the drop is roughly spherical (Fig. 18a). The velocity of the drop remains almost unaltered at the time instant represented in Fig. 18b without noticing the presence of the plate. This reveals that the effect of the immersed wall becomes evident only when the distance from the drop centroid to the wall is very small. The drop lightly tends to acquire an oval shape from the moment where it starts interacting with the wall. The velocity of the drop is dramatically reduced when the drop reaches the wall (Fig. 18c), and eventually changes its direction (Figs. 18d and 18e). At the final stage of the simulation, the velocity of the drop remains close to zero, slightly hovering around this value (Fig. 18f). It is worth noting that the obtained velocity fields are not exactly axisymmetric, causing small lateral deviations. This issue stresses the importance of using a full 3D approach to overcome this problem.

\section{Conclusions}

In the present paper, a coupling between a conservative level set (CLS), a moving mesh (MM) and an immersed boundary (IB) methods has been proposed to address problems of drops and bubbles evolving in complex geometries. First, the CLS method assures a correct representation of the multiphase domain, while the mass error is kept under control [29]. Second, the Arbitrary Lagrangian-Eulerian formulation (i.e. the 


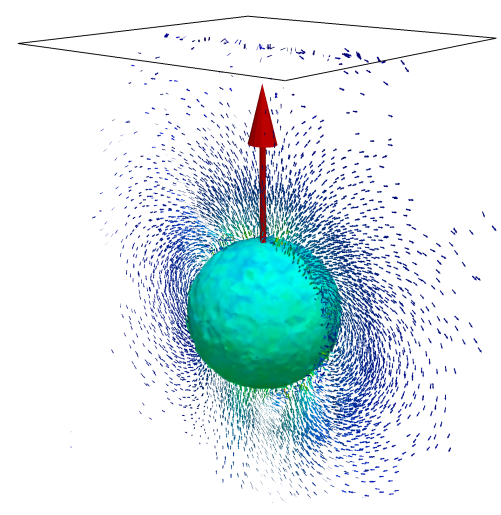

(a)

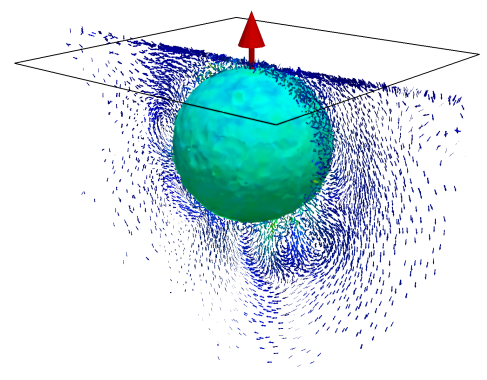

(c)

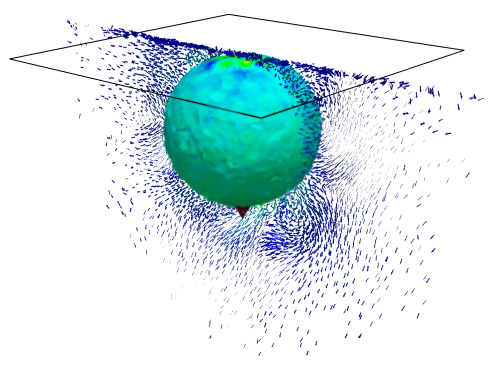

(e)

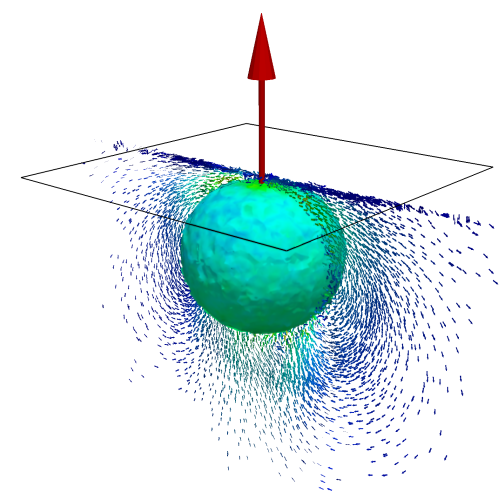

(b)

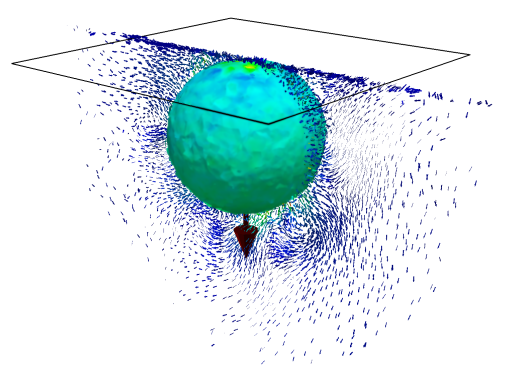

(d)

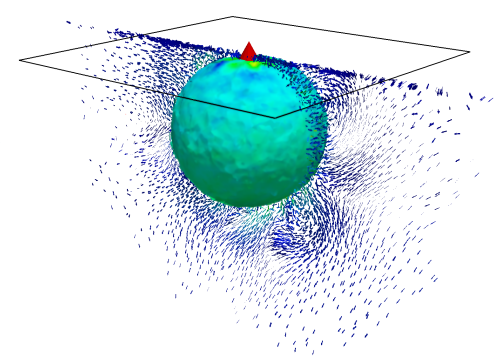

(f)

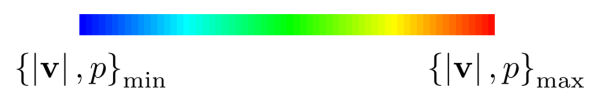

Figure 18: Velocity field and pressure field at different time instants. The pressure field is plotted over the drop surface, and the velocity field is represented in a plane containing the vertical axis. The first image is plotted when the terminal velocity is achieved. Successive images are plotted each $t^{*} \approx 3.1$. The velocity of the centroid of the drop is also represented with a red arrow (not to scale). In these plots, the size of the plate has been reduced to fit the images. 
dynamic mesh) constitutes a robust domain optimization method for bubbles/drops problems [35. It allows the use of a small domain covering only the important regions of the problem, i.e. the current position of the drop/bubble and its surroundings. Otherwise very long domains may be needed in order to leave enough space for the bubble/drop to evolve. Finally, geometrical challenges can be addressed by using the IB method, which constitutes a versatile and robust method to represent intricate solids which do not conform the grid shape.

The proposed method presents some synergetic advantages for the study of bubbles/drops. First, the mesh design process is greatly improved. The control volume sizes are homogenised, and the mesh can be refined at the important regions of the problem as the bubble/drop remains steady within the domain. Moreover, the method greatly facilitates conducting parametric studies, due to the fact that in general there is no need to modify the mesh when a slight variation in the geometry is applied. The IB method also allows to represent solid boundaries within a moving domain. This would otherwise be impossible due to the inherent need of open boundaries (i.e. inflow and outflow) in the moving mesh approach. Finally, it is worth noticing that the use of a framework which supports full unstructured meshes increases the applicability of these features.

Results of the validation cases confirmed the aforementioned ideas. First, a couple of 2D cases were studied in order to be able to compare the results with the same case run with a complete domain. By doing this, differences in the obtained results can be attributed solely to the particularities of the proposed CLS+MM+IB method. Those results indicate a reasonable agreement, with slight differences mainly caused by the presence of the open boundaries in the proposed method. Result comparisons for 3D cases yield a good level of agreement between reference results (mostly experimental) and the ones in the presented method. Apart from the accurateness tests, the result section highlights some important features of the method. First, the differences between simulating a drop/bubble through a non-constricting geometry (cases in Secs. 5.1 and 5.4), and through a constricting one (cases in Secs. 5.2 and 5.3). In particular, the rotational mesh process is clearly illustrated in the problems solved in Secs. 5.1 and 5.2. The capability of solving long physical domains with sort computational domains is plainly stressed in the problem presented in Sec. 5.3 . In this case study, the average results were computed after a significant elapsed computational time. The improvement of the design mesh process is well represented in the problem studied in Sec. 5.4. The region of the bubble and its surroundings are meshed with a high resolution, whereas the regions far from the bubble are coarse-meshed. This adaptability is highlighted throughout all these cases. For example, a change in the inclination of the oblique paths of the problems presented in Secs. 5.1 and 5.2 would only require a simple deformation of the solid. No change in the mesh or code is needed. The versatility of the method also comes into relief when studying the problem presented in Sec. 5.4. In this case study, a rising drop is impacting with an object (a plate). With a classical approach, the distance from the initial drop position to the solid would be a paramount magnitude. It should be reasonably long to assure that the drop reaches the plate at its steady state, but also as short as possible in order to save computational resources. This problem is 
avoided by using the proposed CLS+MM+IB approach. In this case, the drop starts rising freely, and when it is provided that it has achieved its steady motion, the plate is placed at the computational domain limit.

\section{Appendix A Immersed boundary method verification}

To verify the implementation of the chosen IB formulation (Sec. 3.2 , the study of the flow around a sphere for several Reynolds number has been conducted. The flow around a sphere has been subject of several experimental [68, 69, 70, 71, 72] and numerical investigations [72, 73, 174, 75]. The wake behind the sphere becomes increasingly complex as the Reynolds number increases. Based on the mentioned studies, it is known that for $R e \leqslant 200$ the flow remains steady and axisymmetric, for $R e \geqslant 200$ it is still steady but presenting a planar-symmetry structure, over 280 the wake becomes unsteady, and vortices are periodically shed. For $280<R e<400$ the shed vortices have a fixed orientation which produce non-zero mean lift forces. For $R e>400$ the orientation is no longer constant, and the wake becomes asymmetric. Therefore, capturing the dynamics of this flow conditions is a challenging task for non-body conformal grid approaches.

In the present study simulations of the flow around a sphere are performed with Reynolds number of 200, $250,300,350,500$ and 600 .

\section{A.1 Boundary conditions and computational details}

The computational domain is a prism of length $L=25 D$, and height and width $H=W=10 D$, where $D$ is the sphere diameter. Fig. 19 shows this domain configuration. An uniform velocity profile is imposed at the inlet boundary, free-slip conditions are imposed at the lateral walls, and a pressure-based condition is prescribed at the outlet. The sphere is represented as an immersed boundary. It is located at $5 D$ from the inlet, and in the centre of the $y z$-plane.

The domain is discretized using a mesh composed of cubic cells. To obtain a good resolution in the important regions of the problem, and at the same time to minimize the number of cells, three-dimensional local mesh refinement is used (76). The original mesh is formed by 12500 cells of $h=0.2 D$. The cells in the regions indicated in Fig. 19 as $L_{N}$ are refined $N$ times, obtaining cells of sizes equal to $h=0.2 D / 2^{N}$. Inside the boundary layer the cells are refined five times ( $L_{5}$ region). Therefore, the smaller cells have a size of $h=\delta z=0.00625 D$. The final mesh is composed of $1.7 \cdot 10^{6}$ cells.

\section{A.2 Results}

Different cases for the laminar regime have been simulated, varying the Reynolds number between 100 and 600. As expected, for the cases with $R e=100$ and $R e=200$ the steady state and axisymmetry of the flow are observed, which implies a constant drag force and a zero lift force. The flow patterns obtained at 


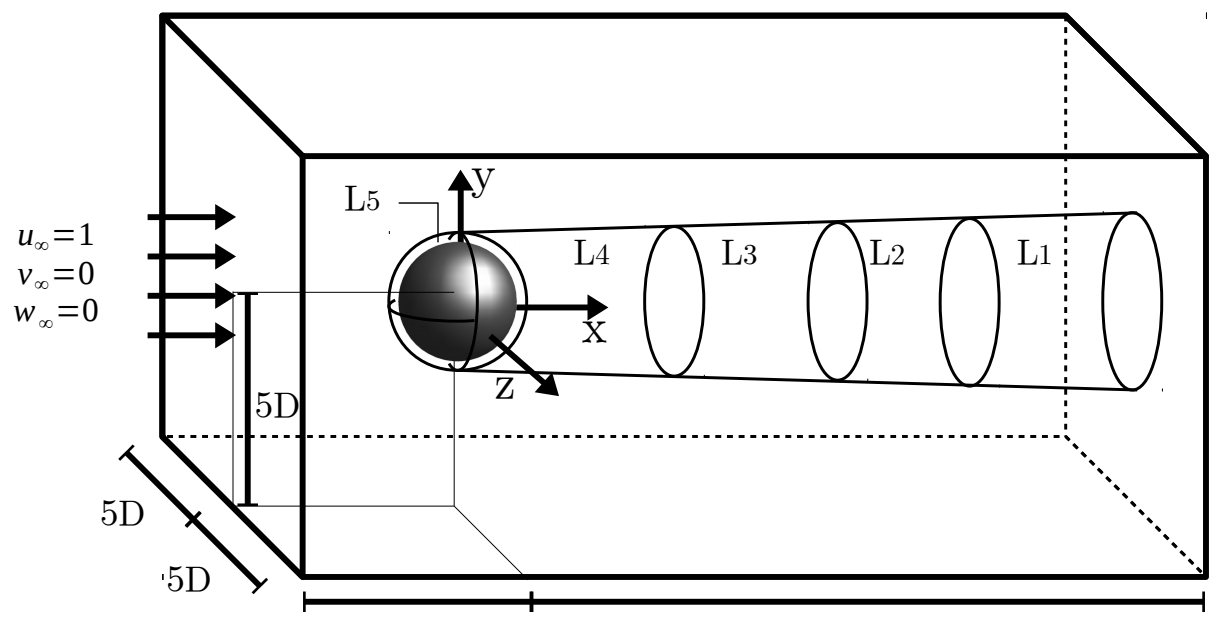

$5 \mathrm{D}$

$20 \mathrm{D}$

Figure 19: Computational domain, boundary conditions and definition of the regions where the local refinement is used.

higher Reynolds numbers are compared against experimental visualizations in Fig. 20" When the Reynolds number is below 420, the expected planar symmetry of the shedding vortices is observed. When that value is overcome, the flow becomes asymmetric. The calculated mean values of $\operatorname{drag}$ and lift coefficients $\left(\bar{C}_{D}\right.$ and $\bar{C}_{L}$ ) are compared against literature results in Fig. 21. Very good agreement was observed. $\bar{C}_{D}$ fits the correlation curve proposed in [77] for all the Reynolds numbers simulated; $\bar{C}_{L}$ is zero for the cases with $R e=100$ and $R e=200$, and positive for the higher Reynolds values. The maximum $\bar{C}_{L}$ is obtained at $R e=300$, as in 75 .

A very different behaviour of the forces in the $y z$-plane is observed in the cases with $R e>200$. These findings are presented in Fig. 22, in the form of a phase diagram $\left(C_{y}, C_{z}\right)$, where the evolution through the dimensionless time $t^{*}=t U / D$ from 50 to 200 is plotted for two Reynolds numbers, 300 and 600 . In the case of $R e=300$, the $y$ and $z$ components of the force $\left(C_{y}, C_{z}\right)$ oscillate with a constant direction, which coincides with the symmetry plane of the vortical structures. Meanwhile, in the case of $R e=600$ the vector $\left(C_{y}, C_{z}\right)$ evolves irregular through time. This is due to the fact that the structures in the wake of the sphere are not planar-symmetric in this regime, showing a disordered behaviour (see Fig. 20.

\footnotetext{
${ }^{2}$ Right column of Fig. 20 was reprinted from Journal of Fluids Engineering, 112:4, H. Sakamoto and H. Haniu, A Study on Vortex Shedding From Spheres in a Uniform Flow, p. 390, Copyright 1990, with permission from the American Society of Mechanical Engineers.
} 


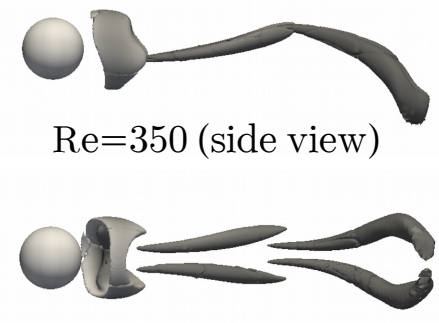

$\mathrm{Re}=350$ (top view)

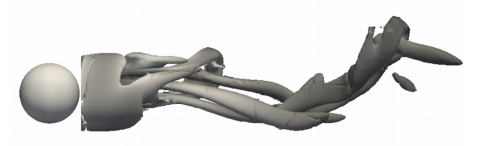

$\operatorname{Re}=600$
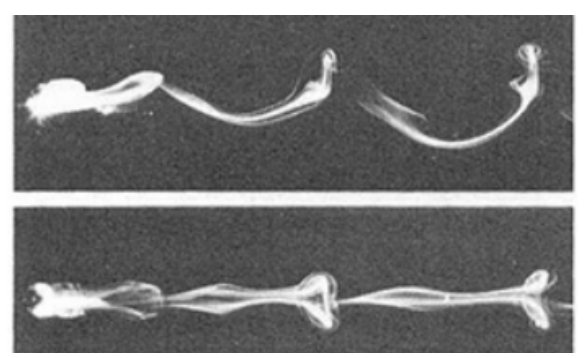

Experiment

$300<\operatorname{Re}<420$

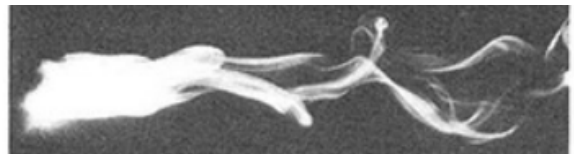

Experiment $420<\operatorname{Re}<800$

Figure 20: Flow patterns of vortex shedding. Comparison between present results: iso-surfaces of the second invariant of the velocity gradient tensor, $\mathrm{Q}$, on the left, and experimental visualization in [71] on the right ${ }^{2}$.

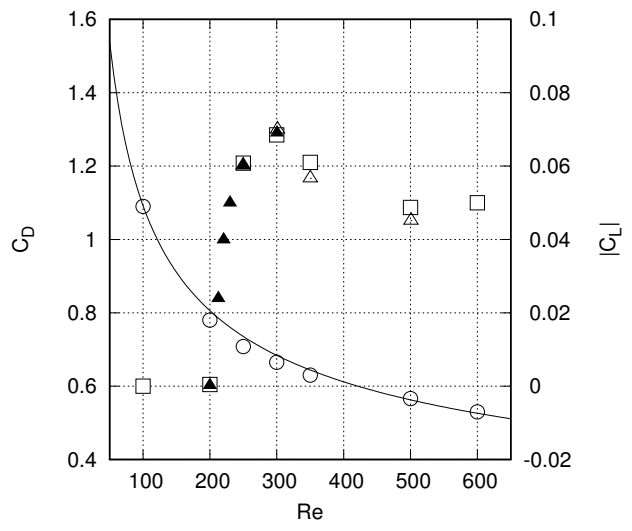

Figure 21: Comparison of present results of time averaged $\bar{C}_{D}$ and $\bar{C}_{L}$ with results from the technical literature. $\bar{C}_{D}$ :

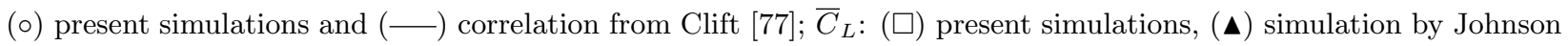
and Pattel 72 and $(\triangle)$ simulation by Bagchi et al. 75].

\section{Appendix B Calculations of bubble/drop properties}

The kinematic properties of a single bubble/drop are computed as explained below. The following expressions are directly given in their discrete form. The position of the bubble/drop centroid $G$ is computed 


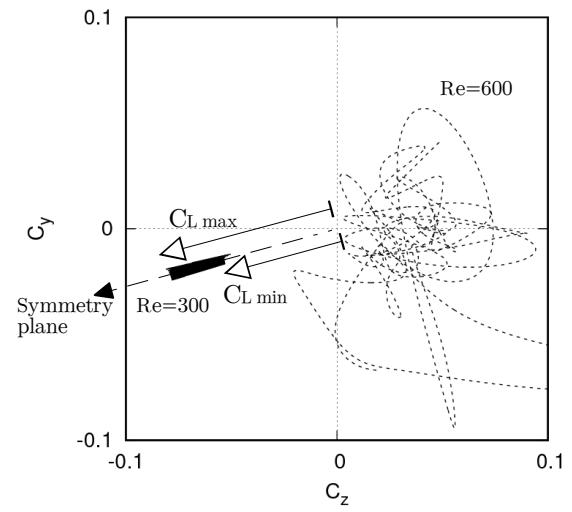

Figure 22: Phase diagram of $\left(C_{y}, C_{z}\right)$ through dimensionless time $t^{*}$ from 50 to 200: $(-) R e=300$ and $(---)$ $R e=600$.

as follows:

$$
\mathbf{x}_{\mathrm{G}}=\frac{\sum_{n} \mathbf{x}^{n} \phi^{n} V^{n}}{\sum_{n} \phi^{n} V^{n}}
$$

where superscript $n$ denotes that the corresponding variable is evaluated at the node $n$ under consideration. $V$ is the volume of the corresponding control volume, $\mathbf{x}$ is the position vector of its centroid, and $\phi$ is the level set function. In addition, the velocity of the bubble/drop is given by:

$$
\mathbf{v}_{\mathrm{G}}=\frac{\sum_{n} \mathbf{v}^{n} \phi^{n} V^{n}}{\sum_{n} \phi^{n} V^{n}}
$$

The total volume of the secondary phase $V_{2}$ can be computed as:

$$
V_{2}=\sum_{n} \phi^{n} V^{n}
$$

Here the integral is computed on the complete domain. Finally, in two dimensional problems, the circularity $\zeta_{2 D}$ measures the deformation with respect to a perfect circular bubble/drop. It is computed as:

$$
\zeta_{2 D}=\pi d \frac{\sum_{n}\|\nabla \phi\|^{n} V^{n}}{\sum_{n} \phi^{n} V^{n}}
$$

where $d$ is the bubble/drop initial diameter. The integral is computed on the complete domain.

\section{Acknowledgments}

The present work has been financially supported by the Ministerio de Economía y Competitividad, Secretaría de Estado de Investigación, Desarrollo e Innovación of Spain (Project ENE-2015-70672P). The authors thankfully acknowledge the computer resources at FinisTerrae2 and technical support provided by $C E S G A$ (RES-FI-2017-2-0042). Calculations have been performed on these facilities and on JFF supercomputer. 


\section{References}

[1] S. Haase, Characterization of gas-liquid two-phase flow in minichannels with co-flowing fluid injection inside the channel, part II: gas bubble and liquid slug lengths, film thickness, and void fraction within Taylor flow, Int. J. Multiph. Flow. 88 (2017) 251-269.

[2] H.A. Stone, A.D. Stroock, A. Ajdari, Engineering flows in small devices: microfluidics toward lab-on-achip. Ann. Rev. Fluid Mech. 36 (2004) 381-411.

[3] G. Tryggvason, R. Scardovelli, S. Zaleski, Direct Numerical Simulations of Gas-Liquid Multiphase Flows, 1st ed. Cambridge University Press, New York, 2011.

[4] M. Hemmat, A. Borhan, Buoyancy-driven motion of drips and bubbles in a periodically constricted capillary, Chem. Eng. Com. 148-150 (1996) 363-384.

[5] A.M. Worthington, On the forms assumed by drops of liquids falling vertically on a horizontal plate. Proc. R. Soc. Lond. 25 (1876) 261-272.

[6] Y. Liu, M. Andrew, J. Li, J. M. Yeomans, Z. Wang, Symmetry breaking in drop bouncing on curved surfaces, Nature Com. 6 (2015).

[7] T. Maitra, C. Antonini, M.K. Tiwari, A. Mularczyk, Z. Imeri, P. Schoch, D. Poulikakos, Supercooled Water Drops Impacting Superhydrophobic Textures, Langmuir 30:36 (2014) 10855-10861.

[8] E. Klaseboer, J.P. Chevaillier, A. Mate, O. Masbernat, C. Gourdon, Model and experiments of a drop impinging on an immersed wall, Phys. Fluids 13:1 (2001) 45-57.

[9] B. Podvin, S. Khoja, F. Moraga, D. Attinger, Model and experimental visualizations of the interaction of a bubble with an inclined wall, Chem. Eng. Sci. 63 (2008) 1914-1928.

[10] N.G. Deen, M. Annaland, J. Kuipers, Direct numerical simulation of complex multi-fluid flows using a combined front tracking and immersed boundary method, Chem. Eng. Sci. 64 (2009) 2186-2201.

[11] A. Gupta, H. Marharoo, D. Makkar, R. Kumar, Droplet formation via squeezing mechanism in a microfluidic flow-focusing device, Comp. \& Fluids 100 (2014) 218-226.

[12] M.W. Baltussen, Q.I.E Segers, J.A.M. Kuipers, N.G. Deen, Cutting bubbles with a single wire, Chem. Eng. Sci. 157 (2017) 138-146.

[13] M. Muradoglu, A.D. Kayaalp, An auxiliary grid method for computations of multiphase flows in complex geometries, J. Comp. Phys., 214 (2006) 858-877. 
[14] J.H. Hills, P. Chéty, The Rising Velocity of Taylor Bubbles in an Annulus, Chem. Eng. Res. \& Des. 76:6 (1998) 723-727.

[15] H.K. Zhao, B. Merriman, S. Osher, L. Wang, Capturing the behavior of bubbles and drops using the variational level set approach, J. Comput. Phys. 143 (1998) 495-518.

[16] I.D. Aleinov, E.G. Puckett, M.M. Sussman, Formation of droplets in microscale jetting devices, Proceedings of FEDSM99 (1999).

[17] U. Olgac, A.D. Kayaalp, M. Muradoglu, Buoyancy-driven motion and breakup of viscous drops in constricted capillaries, Int. J. Multiph. Flow 32:9 (2006) 1055-1071.

[18] S. Protière, M. Z. Bazant, D. A. Weitz, H. A. Stone, Droplet breakup in flow past an obstacle: A capillary instability due to permeability variations, Europhysics Lett., 92:5 (2010) 54002.

[19] M. Roudet, K. Loubiere, C. Gourdon, M. Cabassud, Hydrodynamic and mass transfer in inertial gasliquid flow regimes through straight and meandering millimetric square channels, Chem. Eng. Sci. 66:13 (2011) 2974-2990.

[20] C. Pozrikidis, Passage of a liquid drop through a bifurcation, Eng. Anal. Bound. Elem. 36:2 (2012) 93-103.

[21] S. Misra, B.K. Rana, A.K. Das, P. K. Das, Taylor Bubble Dynamics in Pipe Fittings: A Numerical Study, Fluid Mechanics and Fluid Power - Contemporary Research (2017) 989-1002.

[22] D. Izbassarov, M. Muradoglu, A computational study of two-phase viscoelastic systems in a capillary tube with a sudden contraction/expansion, Phys. Fluids 28:1 (2016).

[23] M.W. Baltussen, J.A.M. Kuipers, N.G. Deen, A numerical study of cutting bubbles with a wire mesh, Chem. Eng. Sci. 165 (2017) 25-32.

[24] G. Tryggvason, B. Bunner, A. Esmaeeli, D. Juric, N. Al-Rawahi, W. Tauber, J. Han, S. Nas, Y.J. Jan, A Front-Tracking Method for the Computations of Multiphase Flow, J. Comput. Phys. 169 (2001) 708-759.

[25] R. Scardovelli, S. Zaleski, Direct numerical simulation of free surface and interfacial flow, Annu. Rev. Fluid Mech. 31 (1999) 567-603.

[26] M. Sussman, E. Fatemi, An efficient interface-preserving level set redistancing algorithm and its application to interfacial incompressible fluid flow, SIAMJ. Sci. Comput. 20 (1999) 1165-1191.

[27] E. Olsson, G. Kreiss, A conservative level set method for two phase flow, J. Comput. Phys. 210 (2005) $225-246$ 
[28] N. Balcázar, O. Lehmkuhl, L. Jofre, J. Rigola, A. Oliva, A coupled volume-of-fluid/level-set method for simulation of two-phase flows on unstructured meshes, Comp. \& Fluids 124 (2016) 12-19.

[29] N. Balcázar, L. Jofre, O. Lehmkuhl, J. Castro, J. Rigola, A finite-volume/level-set method for simulating two-phase flows on unstructured grids, Int. J. Multiph. Flow. 64 (2014) 55-72.

[30] N. Balcázar, O. Lehmkuhl, L. Jofre, A. Oliva, Level-set simulations of buoyancy-driven motion of single and multiple bubbles, Int. J. Heat Fluid Flow. 56 (2015) 91-107.

[31] N. Balcázar, O. Lehmkuhl, J. Rigola, A. Oliva, A multiple marker level-set method for simulation of deformable fluid particles, Int. J. Multiph. Flow. 74 (2015) 125-142.

[32] M. Chai, K. Luo, C. Shao, J. Fan, An efficient level set remedy approach for simulations of two-phase flow based on sigmoid function, Chem. Eng. Sci. 172 (2017) 335-352.

[33] O. Desjardins, V. Moureau, H. Pitsch, An accurate conservative level set/ghost fluid method for simulating turbulent atomization, J. Comput. Phys. 227 (2008) 8395-8416

[34] N. Balcázar, J. Castro, J. Rigola, A. Oliva, DNS of the wall effect on the motion of bubble swarms. Procedia Comput. Sci. 108 (2017) 2008-2017.

[35] E. Gutiérrez, N. Balcázar, E. Bartrons, J. Rigola, Numerical study of Taylor bubbles rising in a stagnant liquid using a Level-set / Moving-Mesh method, Chem. Eng. Sci. 164 (2017) 158-177.

[36] O. Estruch, O. Lehmkuhl, R. Borrell, C. D. Pérez Segarra, A. Oliva, A parallel radial basis function interpolation method for unstructured dynamic meshes, Comput. Fluids. 80 (2013) 44-54.

[37] R. Mittal, G. Iaccarino, Immersed Boundary Methods. Annual Review of Fluid Mechanics, 37:1 (2005) 239-261.

[38] C.S. Peskin, Flow patterns around heart valves: a numerical method, J. Comput. Phys., 10 (1972) $252-271$.

[39] C.S. Peskin, D.M. McQueen, Modeling prosthetic heart valves for numerical analysis of blood flow in the heart, J. Comput. Phys., 37:1 (1980) 113-132.

[40] C.S. Peskin, D.M. McQueen, Cardiac fluid dynamics, Crit. Rev. Biomed. Eng., 20:5-6 (1997) 451-459.

[41] D.M. McQueen, C. S. Peskin, Shared-Memory Parallel Vector Implementation of the Immersed Boundary Method for the Computation of Blood Flow in the Beating Mammalian Heart, J. Supercomput., 236 (1997) 213-236. 
[42] M.C. Lai, C.S. Peskin. An immersed boundary method with formal second-order accuracy and reduced numerical viscosity, J. Comput. Phys., 160:2 (2000) 705-719.

[43] J. Mohd-Yusof, Development of immersed boundary methods for complex geometries, Center for Turbulence Research, Annual Research Briefs, 1997, 325-336.

[44] E.A. Fadlun, R. Verzicco, P. Orlandi, J. Mohd-Yusof. Combined immersed-boundary finite-difference methods for three-dimensional complex flow simulations, J. Comput. Phys., 161:1 (2000) 35-60.

[45] E. Balaras, Modeling complex boundaries using an external force field on fixed Cartesian grids in largeeddy simulations, Comp. \& Fluids, 33:3 (2004) 375-404.

[46] K. Nagendra, D.K. Tafti, K. Viswanath, A new approach for conjugate heat transfer problems using immersed boundary method for curvilinear grid based solvers, J. Comput. Phys., 267 (2014) 225-246.

[47] Y.H. Tseng, J.H. Ferziger, A ghost-cell immersed boundary method for flow in complex geometry, J. Comput. Phys., 192:2 (2003) 593-623.

[48] K. Luo, Z. Zhuang, J. Fan, N. Erland, L. Haugen, A ghost-cell immersed boundary method for simulations of heat transfer in compressible flows under different boundary conditions, Int. J. Heat Mass Transfer, Volume 104, 2017, Pages 98-111.

[49] G. Yang, D. M. Causon, D. M. Ingram. Calculation of compressible flows about complex moving geometries using a three-dimensional Cartesian cut cell method. Int. J. Numer. Methods Fluids., 33:8 (2000) 1121-1151.

[50] H.S. Udaykumar, R. Mittal, P. Rampunggoon, A. Khanna, A sharp interface Cartesian grid method for simulating flows with complex moving boundaries, J. Comput. Phys. 174:1 (2001) 345-380.

[51] T. Ye, R. Mittal, H.S. Udaykumar, W. Shyy. An Accurate Cartesian Grid Method for Viscous Incompressible Flows with Complex Immersed Boundaries, J. Comput. Phys., 156:2 (1999) 209-240.

[52] J.H. Seo, R. Mittal, A sharp-interface immersed boundary method with improved mass conservation and reduced spurious pressure oscillations, J. Comput. Phys., 230:19 (2011) 7347-7363.

[53] A. Harten, The artificial compression method for computation of shocks and contact discontinuities. III - Self-adjusting hybrid schemes, Math. Comp. 32 (1978) 363-389.

[54] J.U. Brackbill, D.B. Kothe, C. Zemach, A continuum method for modeling surface tension, J. Comput. Phys. 100 (1992) 335-354.

[55] S. Gottlieb, C. Shu, Total variation diminishing Runge-Kutta schemes, Math. Comp. 67 (1998) 73-85. 
[56] A.J. Chorin. Numerical solution of the Navier-Stokes equations, Math. Comp. 22 (1968) 745-762.

[57] J.L. Guermond, P. Minev, J. Shen, An overview of projection methods for incompressible flows, Comput. Methods Appl. Mech. Eng. 195 (2006) 6011-6045.

[58] N. Balcázar, J. Rigola, J. Castro, A. Oliva, A level-set model for thermocapillary motion of deformable fluid particles, Int. J. Heat Fluid Flow. 62B (2016) 324-343.

[59] R.W. Davis, E.F. Moore, A numerical study of vortex shedding from rectangles, J. Fluid Mech. 116 (1982) 475-506.

[60] Wikipedia: STL (file format) https://en.wikipedia.org/wiki/STL_(file_format) (accessed 8 January 2018).

[61] F. Favre, G. Colomer, O. Lehmkuhl, A. Oliva, Numerical simulations of conjugate convection combined with surface thermal radiation using an Immersed-Boundary Method, J. Phys. Conf. Ser. 745:3 (2016).

[62] R.W.C.P. Verstappen, A.E.P. Veldman, Spectro-consistent discretization of Navier-Stokes: a challenge to RANS and LES, J. Engrg. Math. 34 (1998) 163-179.

[63] M. Meyer, A. Devesa, S. Hickel, X. Y. Hu, N. A. Adams. A conservative immersed interface method for Large-Eddy Simulation of incompressible flows. J. Comput. Phys., 229:18 (2010) 6300-6317.

[64] Termo Fluids S.L. http://www.termofluids.com/, (accessed 8 January 2018).

[65] S. Hysing, S. Turek, D. Kuzmin, N. Parolini, E. Burman, S. Ganesan, L. Tobiska, Quantitative benchmark computations of two-dimensional bubble dynamics, Int. J. Numer. Meth. Fluids 60 (2009) 12591288.

[66] J.D. Bugg, G.A. Saad, The velocity field around a Taylor bubble rising in a stagnant viscous fluid: Numerical and experimental results, Int. J. Multiph. Flow. 28 (2002) 791-803.

[67] T.Z. Harmathy, Velocity of large drops and bubbles in media of infinite or restricted extent, AIChE J. 6 (1960) 281-288.

[68] Elmar Achenbach. Experiments on the flow past spheres at very high Reynolds numbers, J. Fluid Mech. $54: 3$ (1972) 565-575.

[69] Elmar Achenbach, Vortex shedding from spheres, J. Fluid Mech. 62:2 (1974), 209.

[70] H. J. Kim, P. A. Durbin, Observations of the frequencies in a sphere wake and of drag increase by acoustic excitation, Phys. Fluids. 31:11 (1988), 3260. 
[71] H. Sakamoto, H. Haniu, A Study on Vortex Shedding From Spheres in a Uniform Flow, J. Fluids Eng. 112:4 (1990), 386.

[72] T. A. Johnson, V. C. Patel, Flow past a sphere up to a Reynolds number of 300, J. Fluid Mech. 378 (1999) 19-70.

[73] R. Mittal, F. Najjar, Vortex dynamics in the sphere wake. 30th Fluid Dynamics Conference (1999) 1-8.

[74] A. G. Tomboulides, S. Orszag, Numerical investigation of transitional and weak turbulent flow past a sphere. J. Fluid Mech. 416 (2000) 45-73.

[75] P. Bagchi, M. Y. Ha, S. Balachandar, Direct Numerical Simulation of Flow and Heat Transfer From a Sphere in a Uniform Cross-Flow. J. Fluids Eng. 123:2 (2001) 347.

[76] O. Antepara, O. Lehmkhul, R. Borrell, J. Chiva, A. Oliva, Parallel adaptive mesh refinement for largeeddy simulations of turbulent flows, Comp. \& Fluids 110 (2015) 48-61.

[77] R. Clift, Bubbles, Drops and Particles, volume 94. Academic Press, Inc., New York, 1979. 